\title{
Comparisons of protein profiles of beech bark disease resistant and susceptible American beech (Fagus grandifolia)
}

Mary E Mason ${ }^{1}$, Jennifer $L$ Koch $^{2 *}$, Marek Krasowski ${ }^{3}$ and Judy Loo ${ }^{4,5}$

\begin{abstract}
Background: Beech bark disease is an insect-fungus complex that damages and often kills American beech trees and has major ecological and economic impacts on forests of the northeastern United States and southeastern Canadian forests. The disease begins when exotic beech scale insects feed on the bark of trees, and is followed by infection of damaged bark tissues by one of the Neonectria species of fungi. Proteomic analysis was conducted of beech bark proteins from diseased trees and healthy trees in areas heavily infested with beech bark disease. All of the diseased trees had signs of Neonectria infection such as cankers or fruiting bodies. In previous tests reported elsewhere, all of the diseased trees were demonstrated to be susceptible to the scale insect and all of the healthy trees were demonstrated to be resistant to the scale insect. Sixteen trees were sampled from eight geographically isolated stands, the sample consisting of 10 healthy (scale-resistant) and 6 diseased/infested (scale-susceptible) trees.

Results: Proteins were extracted from each tree and analysed in triplicate by isoelectric focusing followed by denaturing gel electrophoresis. Gels were stained and protein spots identified and intensity quantified, then a statistical model was fit to identify significant differences between trees. A subset of BBD differential proteins were analysed by mass spectrometry and matched to known protein sequences for identification. Identified proteins had homology to stress, insect, and pathogen related proteins in other plant systems. Protein spots significantly different in diseased and healthy trees having no stand or disease-by-stand interaction effects were identified.

Conclusions: Further study of these proteins should help to understand processes critical to resistance to beech bark disease and to develop biomarkers for use in tree breeding programs and for the selection of resistant trees prior to or in early stages of BBD development in stands. Early identification of resistant trees (prior to the full disease development in an area) will allow forest management through the removal of susceptible trees and their root-sprouts prior to the onset of disease, allowing management and mitigation of costs, economic impact, and impacts on ecological systems and services.
\end{abstract}

Keywords: Beech bark disease, Beech scale, Disease resistance, Insect resistance, Fagus, Cryptococcus, Neonectria

\section{Background}

Beech bark disease (BBD) is an insect-fungus complex that has been killing American beech (Fagus grandifolia Ehrh.) trees since the accidental introduction of the beech scale insect (Cryptococcus fagisuga Lind.) to Canada around $1890[1,2]$. The first phase of BBD is beech scale insect infestation resulting in the production of small fissures in the bark [1]. The fungal component,

\footnotetext{
* Correspondence: jkoch@fs.fed.us

US Forest Service, Northern Research Station, 359 Main Rd, Delaware, $\mathrm{OH}$ 43015, USA

Full list of author information is available at the end of the article
}

either Neonectria ditissima Samuels \& Rossman or Neonectria faginata Castlebury then infects these fissures causing extensive tissue damage. Mortality in the first wave of the disease can be as high as $50 \%$ [3], with consequent loss to stand health, merchantable timber, and many wildlife and ecosystem services.

An estimated 1\% of American beech trees remain disease free in forests long-affected by beech bark disease [4]. Insect challenge experiments have demonstrated that resistance is to the beech scale portion of the disease complex $[4,5]$. Although it has been reported that scale infestation without Neonectria infection may play a

\section{Biomed Central}


role in mortality events [6], there has been no documentation of Neonectria infection leading to widespread stand or landscape level mortality in the absence of prior scale infestation. In aftermath forests, where scale populations have declined, presumably due to loss or reduced quality of habitat [7] and environmental factors [8], the population dynamics of scale and Neonectria are no longer directly correlated $[7,9]$. However, in these cases it is suggested that the lower density of scale is still sufficiently high that infection sites (scale-feeding wounds) are not limiting and once the fungus has established in the tree it is no longer influenced by fluctuations in scale density [7]. Therefore, the focus of recent breeding and tree improvement efforts has been on resistance to the scale insect $[5,10]$. Current management approaches are based on the objective of increasing the proportion of disease resistant beech by removing susceptible trees along with any resulting root and stump sprouts, retaining the disease-free trees prior to the height of BBD development $[11,12]$ and supplementing with genetically scale-resistant seedling plantings once such materials are available (management plans of US Forest Service Allegheny National Forest [13] and Michigan Dept. of Natural Resources [14]). However, it is impossible to identify the most resistant beech trees until the scale infestation is heavy, at which point economic losses have already occurred and management operations are more complicated and expensive. Identification of a biomarker for Cryptococcus resistance would provide land managers the opportunity to begin management operations before the economic and ecological losses have occurred, and to spread BBD management activities over several budget cycles. A biomarker for resistance may also be utilized to expedite the breeding and selection process.

Bark protein differences are likely to be a good source for biomarker candidates. The scale insect feeds in the tissue layers vernacularly known as bark (cork, cork cambium, phloem, and cambium)[1]. Wargo et al. [15] found significant differences in bark amino acid concentrations and total amino nitrogen in different seasons and scale infestation levels. These results suggest bark protein content may be important in the insect-tree interaction, and that bark protein profiles may differ between scale-resistant and susceptible beech trees. Therefore differentially expressed bark proteins may be reliable biomarkers of resistance to beech scale in American beech. One method to identify differentially expressed bark proteins is to examine the proteome of a number of trees using two dimensional electrophoresis (2-DE) gels. A proteomics approach allows the examination and quantification of large numbers of proteins anonymously and simultaneously. Typically, 2-DE analysis is limited to two-sample comparison with simple experimental structure (e.g. one treatment). Utilization of analysis of variance (ANOVA) for statistical analysis allows the testing of three or more treatment levels for several technical and biological factors in one model, and supports unbalanced experimental and sampling designs. This more sophisticated analysis allows the identification of more complex protein quantity patterns and the interactions of factors in protein quantity. In this study we employ the use of 2-DE gel based proteomics and ANOVA to identify proteins in the bark of American beech that are different between healthy and BBD diseased trees, while also considering if the BBD effect is present alone or with a stand effect or interaction between stand and BBD effects. Although the healthy trees in this study are known to be resistant to the scale insect, the diseased trees are susceptible to both scale infestation (Table 1), and had symptoms of an active fungal infection at the time of tissue collection. Proteins that are expressed in response to the scale insect cannot be distinguished from proteins expressed in response to the fungal pathogen in diseased trees so in our analysis we refer to the more general BBD response which includes responses to both. This approach allows selection of proteins for further study that are most likely to be broadly linked to BBD response rather than different in protein quantity due to the relatedness of trees within stands.

\section{Results}

\section{Individual tree analysis and spot matching}

The location, field disease score, and the artificial infestation (scale resistant/scale susceptible) results for the ten healthy trees and six diseased trees studied are summarized in Table 1. Artificial infestation of grafted ramets of the healthy trees demonstrated that all of these genotypes are resistant to the scale insect, the details of these experiments are reported elsewhere [16]. Protein was extracted and 2-DE was conducted for three technical replicates per tree. Figure 1 shows a randomly selected experimental gel to illustrate the typical resolution and spot density we achieved in the experiment. PDQuest was used to create a master gel for each tree and the number of protein spots per tree ranged from 197 to 522 with an average of 305.3 and standard error of 23 .

An experiment-wide master gel was constructed using the 'compare experiments' function of PDQuest where each individual tree master was considered an 'experiment'. The experiment wide master gel (Figure 2) included all spots on individual tree masters that were added to the experiment wide master because they were present in two or more trees. Most, but not all, of these spots were present in more than two trees, and some spots were present in all trees. The number of matched and unique spots for each tree is listed in Table 1. The total number of spots added to the experiment wide 
Table 1 Tree information including stand, field disease score, controlled test insect challenge result, and spot quantities

\begin{tabular}{|c|c|c|c|c|c|c|}
\hline Tree name & Stand code ${ }^{a}$ & Disease Condition $^{\mathbf{b}}$ & Scale Interaction ${ }^{c}$ & Total protein spots & Number of matched spots & Number of unique spots \\
\hline 1504 & LM & $\mathrm{H}$ & $\mathrm{R}(8)$ & 382 & 295 & 87 \\
\hline 1506 & LM & $\mathrm{D}$ & S (3) & 197 & 184 & 13 \\
\hline $\mathrm{CLO}^{\mathrm{d}}$ & $C L$ & $\mathrm{H}$ & $R(10)$ & 258 & 241 & 17 \\
\hline CM01 & CM & $\mathrm{H}$ & $\mathrm{R}(1)$ & 280 & 270 & 10 \\
\hline$C M 02 D^{d}$ & CM & $\mathrm{D}$ & $S(11)$ & 329 & 329 & 0 \\
\hline DTO2 $^{d}$ & DT & $\mathrm{H}$ & $\mathrm{R}(2)$ & 228 & 215 & 13 \\
\hline DT02D & DT & $\mathrm{D}$ & not tested & 286 & 266 & 20 \\
\hline DTOA $^{d}$ & DT & $\mathrm{H}$ & $\mathrm{R}(2)$ & 522 & 421 & 101 \\
\hline $\mathrm{JCO}^{\mathrm{d}}$ & $J C$ & $\mathrm{H}$ & $\mathrm{R}(14)$ & 367 & 338 & 29 \\
\hline$J C 02 D$ & $J C$ & $\mathrm{D}$ & S (4) & 487 & 408 & 79 \\
\hline $\mathrm{OD} 02^{\mathrm{d}}$ & OD & $\mathrm{H}$ & $R(10)$ & 284 & 272 & 12 \\
\hline OD08D ${ }^{d}$ & OD & $\mathrm{D}$ & $S(12)$ & 239 & 225 & 14 \\
\hline $\mathrm{RSO}^{\mathrm{d}}$ & RS & $\mathrm{H}$ & $\mathrm{R}(9)$ & 288 & 265 & 23 \\
\hline RS02D & RS & D & $S(7)$ & 229 & 223 & 6 \\
\hline$S L 01^{d}$ & $S L$ & $\mathrm{H}$ & R (4) & 249 & 240 & 15 \\
\hline $\mathrm{SLO}^{\mathrm{d}}$ & $S L$ & $\mathrm{H}$ & R (8) & 260 & 243 & 17 \\
\hline
\end{tabular}

${ }^{a} \mathrm{LM}=$ Ludington, MI, USA; CL= Charlie Lake, NB, Can; CM= Curry Mountain, NB, Can; DT= Roachville, NB, Can; JC=Highfield, NB, Can; OD= Odell Park, Fredericton, $\mathrm{NB}, \mathrm{Can} ; \mathrm{RS}=$ Regent St., Fredericton, NB, Can; SL=Spednick Lake, NB, Can.

${ }^{\mathrm{b}} \mathrm{H}=$ Healthy (lacking scale infestation and signs of fungal infection), $\mathrm{D}=$ Diseased (beech bark disease present both scale infestation and fungal infection observed (on ortet) in the field).

'Test result of artificial inoculation of grafted ramets with scale insect eggs. Result (number of ramets tested, i.e. replicates). $\mathrm{R}=\mathrm{Resistant,0-5}$ avg. live scale, $\mathrm{S}=$ Susceptible, 30-100 avg. live scale and expected to sustain scale population and subsequent fungal infection.

${ }^{\mathrm{d}}$ Correspondence to Ramirez et al. [16] CL01=E1, CM02d=H1, DT02=B3, DT0A=B1, JC01=C1, OD02=A2, OD08d=A3, RS01=I1, SL01=D1, and SL03=D3.

master (matched in two or more trees) was 531. Matched spot per tree ranged from 184 to 421 and average $277.2+/-16.7$. Unique spots per tree (spots in the tree master not matched and added to the experiment wide master) range from 0 (base tree CM02d has all spots on the master gel by definition) to 101 with an average of $28.5+/-7.75$. The grand total of spots identified is 987 which is the sum of the 531 matched spots plus 456 spots unique to only one tree. Spots unique to a single tree were excluded from further analysis. While unique spots in aggregate are $46 \%$ of total spots, a maximum of $22.7 \%$ of the spots in any one tree are unique indicating that the spot matching of trees to the experiment wide master was efficient.

Figure 1 Raw gel image of one randomly selected gel. Gel image from tree DT02, shown to illustrate the number of spots and quality of separation typical of the experimental gels. The gel has been cropped to remove gel edge and markers, but include both pl and electrophoresis fronts. 


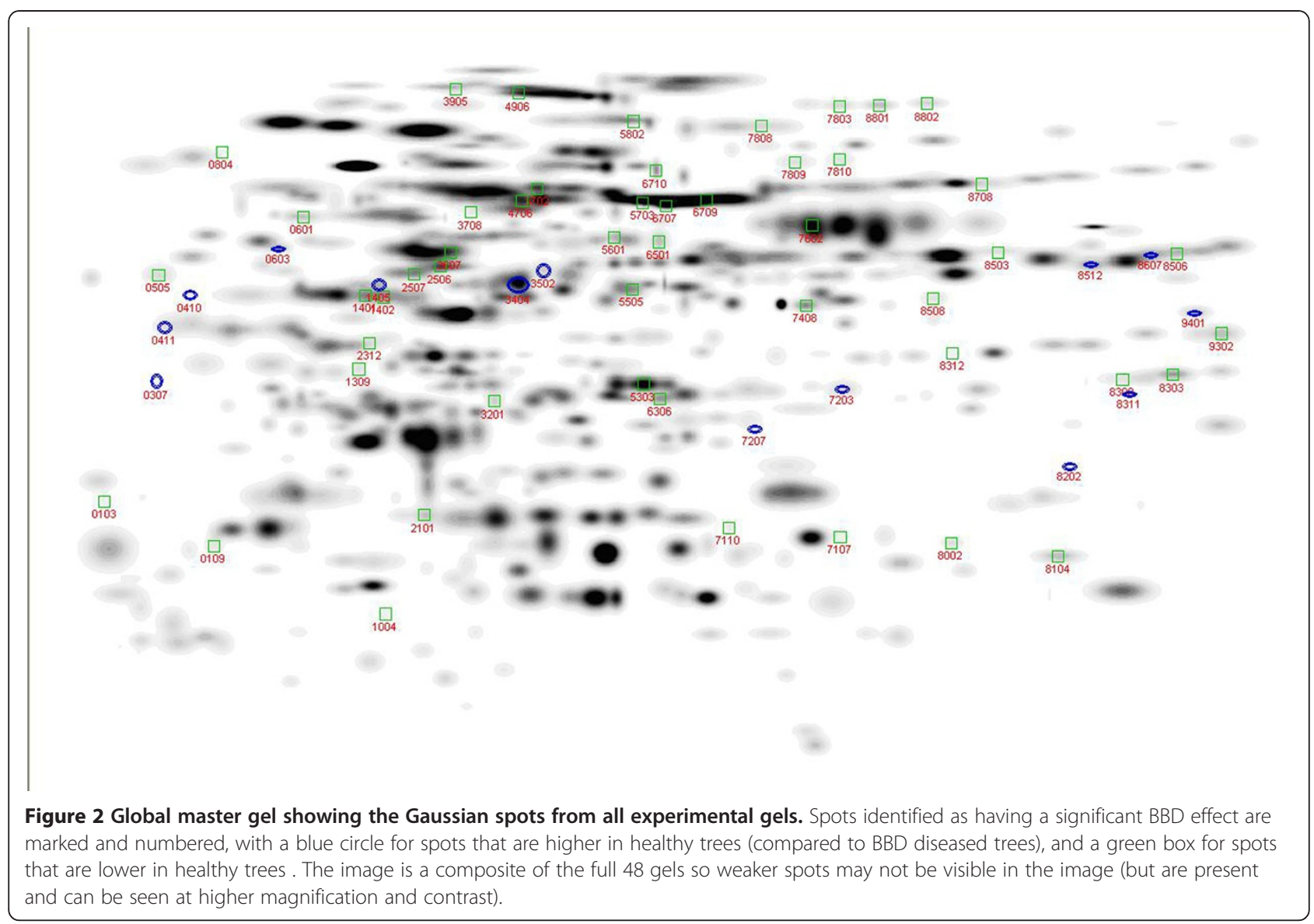

To consider whether these unique spots could be artifacts due to low spot intensity, four trees were selected at random and the spot intensity distribution examined. Graphing showed that the distribution of unique spots was slightly biased toward lighter spots. However, comparison of summary statistics (mean, min, Q1, mean, Q3, max) illustrates that spots of similar intensity are both matched and unmatched, and the faintest spot is matched in three of the four trees (data not shown). This indicates that the spots unique to each tree are not artifacts of poor matching related to the intensity of the spots or variances in protein quantification. Spot quantities for all matched spots in all trees were exported for analysis (missing spots were estimated) in order to use more robust statistical procedures than PDQuest allows.

\section{Constitutive proteins and technical effects}

General linear models were fitted to assess whether technical effects (gel and batch) were significant or could be ignored, and to identify constitutive proteins (spot quantity equal in all individual trees, tested by fitting a tree effect). Technical effects were significant for only six spots and these spots were dropped from further analysis, allowing technical effects to be dropped from later models. The biological effects of interest are stand and disease state, and constitutive spots will obviously not vary for these effects. Therefore the 103 constitutive spots (tree effect not significant) were removed from the dataset to reduce its size for more efficient analysis (leaving 422 spots that had a significantly different mean spot quantity in at least one tree and no significant technical effects).

\section{Stand and disease condition effects}

General linear models were fitted to test for disease condition effect (BBD), stand (STAND) and the disease condition by stand interaction (INT) for the retained spots. The count of spots significant for different combinations of effects is shown in Table 2. The largest class of proteins contains those that are quantitatively different in several trees (demonstrated in the first model, but not significant for any tested effects (209 spots)). Protein spots with a significant STAND effect but no significance for BBD (61 spots of which 28 have a significant interaction and 33 do not) are interesting as potential markers for geographic variation in beech. These spots and an additional 32 spots with only the interaction effect found significant were not studied further in this experiment. 
Table 2 Significant protein counts

\begin{tabular}{llllll}
\hline & \multicolumn{3}{c}{ Stand x BBD Interaction } \\
\cline { 2 - 3 } \cline { 6 - 6 } \cline { 5 - 6 } STAND effect & \multicolumn{3}{c}{ Significant } & & Not Significant \\
\cline { 2 - 3 } \cline { 5 - 6 } & Significant & Not Sig. & & Significant & Not Sig. \\
\hline BBD Effect Significant & 47 & 9 & & 14 & 50 \\
BBD Effect Not & 28 & 32 & & 33 & 209 \\
Significant & & & & \\
\hline
\end{tabular}

Count of proteins with significant effects for stand, beech bark disease state, and stand $x$ disease state interaction. The most likely biomarker candidates are the protein spots with a significant BBD effect in the absence of stand effects and interactions. To see the correspondence to Figure 3 may require summing across cells, for example the 56 spots where the interaction and BBD effect are significant is the sum of the two stand levels, 47 plus nine.

Table 3 shows the protein spots with significant BBD effect, including the p-value and q-value for the BBD effect, the mean spot quantity and standard error for the diseased and healthy trees, and the ratio and direction of differences of spot quantity of healthy to diseased to trees (for all p-values underlying Table 3, see Additional file 1). One-hundred and twenty spots have a significant BBD effect, and of these 50 have no other significant effects and are the most logical candidates for biomarker development (marked with an asterisk in Table 3).

\section{Spot selection and LC-MS/MS analysis}

Spots were selected for coring and sequencing based upon the BBD effect being significant (including some with other significant effects as well) and the location of the spot in the gel being conducive to excising a clean gel core (only one protein in the core, some areas were too densely packed to allow coring). The trees 1504 and CM02d were chosen for use in preparative gels because these trees contained the most proteins of interest (33 and 26 respectively). Attempts were made to sample all BBD significant spots in these trees, and images were carefully evaluated after spot cutting to verify the intended spot was recovered for analysis. In addition to the spots of interest, several well isolated spots were cored for quality control purposes. A total of 28 gel spots (20 BBD significant, 4 control, and 4 cut from both gels) were successfully recovered and analysed by LCMS/MS. Of the 50 highest interest spots, 15 were successfully cored and sequenced. Resulting peptide spectra were identified by matching to NCBI datasets, or in a 2phase matching strategy matched to beech EST's that were then matched to NCBI sequences. Of the 28 spots sequenced, 20 were identified (highest scoring matches or ties only, Table 4) based upon homology to known plant sequences, or homology of the matched EST to plant sequences (including all four control spots). Of the 15 sequenced from the 50 highest interest spots, 11 were identified by sequence homology. There are a few cases where spots were matched to more than one significant identification (spots 307, 2101, and 7408), but in two of them identical peptides returned multiple database entries with different annotations (spots 307 and 2101). The use of the EST database in spot identification greatly improved the success rate at identifying proteins, as over half of the identifications were made using the EST database and would have been unidentified had only Genbank been used. The majority of the spots that were identified based on sequence homology have been shown to be stress-related in other plant systems (Table 5).

\section{Utility of the analysis to narrow the biomarker candidate pool}

In order to illustrate the discriminatory power of our approach we have illustrated the spot set reduction strategy in Figure 3. Beginning with the 987 total protein spots identified, we show how at each step some spots are discarded from further consideration as a biomarker. The final set for continued biomarker considerations is eleven spots that have a BBD effect only (not confounded by stand effects or interactions between BBD and STAND) and are identified by their sequence homology.

\section{Discussion}

\section{Challenges of proteomic investigation of forest trees}

In general, protein extraction from plant tissue is technically challenging due to the high proportion of contaminants relative to the low concentration of protein. Proteomics in forest trees is further complicated by the complexity of working with trees as an experimental system due to factors such as their large size, long life cycle, and large genome [51]. In contrast to most proteomics studies conducted on model organisms, our subjects are wild, unrelated, mature trees selected from multiple stands. Like many forest trees, American beech is windpollinated and has a low self-pollination rate [52], resulting in high heterozygosity among trees within stands [53]. We selected trees from eight non-contiguous stands, further decreasing any chance of relatedness between trees across the study and likely increasing the number of alleles per locus sampled [54]. These factors lead to our study having a much higher degree of genetic complexity in the sampling units than is generally encountered in proteomics work where the use of inbred lines, clones, or pooling across genotypes is common. In addition, the multi-component nature of beech bark disease also adds to the complexity of protein patterns. Due to BBD having both an insect and a fungal component, both wound/insect and pathogen responsive genes are likely to be detected in diseased trees. In addition, BBD develops over a time scale of months or years, rather than the time course of days often studied in wound (insect), gene-for-gene, or viral pathosystems. BBD develops as a 
Table 3 Significant effects, means, and expression ratio for protein spots with significant beech bark disease (BBD) effect

\begin{tabular}{|c|c|c|c|c|c|c|c|c|c|c|}
\hline Spot & & BBD q-value ${ }^{a}$ & STAND $^{\mathbf{b}}$ & $\mathrm{INT}^{\mathrm{C}}$ & mean_H $H^{d}$ & sd_H $\mathrm{H}^{\mathrm{d}}$ & mean_Dd & sd_D ${ }^{d}$ & Ratio of means ${ }^{e}$ & Direction in healthy \\
\hline 11 & & 0.0470 & NS & SIG & 26,174 & 65,030 & 72,775 & 87,910 & 2.7804 & down \\
\hline 13 & & 0.0328 & SIG & SIG & 94,894 & 219,401 & 59,736 & 143,489 & 1.5886 & up \\
\hline 19 & & $0.0000^{f}$ & SIG & SIG & 8,004 & 5,915 & 62,232 & 97,523 & 7.7749 & down \\
\hline 103 & * & 0.0199 & NS & NS & 55,653 & 177,669 & 296,935 & 581,023 & 5.3354 & down \\
\hline 108 & & 0.0004 & SIG & SIG & 258,261 & 376,114 & 111,723 & 168,407 & 2.3116 & up \\
\hline 109 & * & 0.0023 & NS & NS & 617,595 & 986,852 & 153,958 & 248,739 & 4.0114 & up \\
\hline 110 & & 0.0001 & SIG & SIG & 39,356 & 97,166 & 220,462 & 387,308 & 5.6017 & down \\
\hline 210 & & 0.0004 & NS & SIG & 757,282 & 605,616 & 285,096 & 446,196 & 2.6562 & up \\
\hline 303 & & 0.0002 & SIG & SIG & 173,237 & 616,608 & 633,878 & 976,099 & 3.6590 & down \\
\hline 306 & & 0.0022 & NS & SIG & 8,004 & 5,915 & 149,834 & 274,377 & 18.7195 & down \\
\hline 307 & & 0.0275 & SIG & NS & 759,264 & 688,274 & 302,438 & 516,028 & 2.5105 & up \\
\hline 408 & & $0.0000^{f}$ & SIG & SIG & 258,525 & 539,218 & 856,239 & $1,833,144$ & 3.3120 & down \\
\hline 410 & & 0.0041 & SIG & NS & 26,580 & 63,389 & 118,326 & 141,769 & 4.4517 & down \\
\hline 411 & & 0.0295 & SIG & NS & 165,226 & 431,575 & 708,030 & $1,274,849$ & 4.2852 & down \\
\hline 412 & & 0.0054 & SIG & SIG & 447,495 & $1,004,238$ & 310,856 & 722,114 & 1.4396 & up \\
\hline 505 & * & 0.0470 & NS & NS & 925,741 & 886,816 & 477,691 & 544,289 & 1.9379 & up \\
\hline 601 & * & 0.0318 & NS & NS & 333,862 & 424,610 & 725,308 & 843,336 & 2.1725 & down \\
\hline 602 & & $0.0000^{f}$ & SIG & SIG & 365,242 & 873,890 & $1,394,639$ & $1,721,661$ & 3.8184 & down \\
\hline 603 & & 0.0076 & SIG & NS & $1,533,077$ & $1,657,207$ & $1,053,773$ & $1,360,574$ & 1.4548 & up \\
\hline 606 & & 0.0216 & SIG & $\mathrm{SIG}$ & 43,523 & 126,690 & 8,417 & 8,283 & 5.1708 & up \\
\hline 610 & & 0.0001 & SIG & SIG & 146,957 & 447,440 & 8,417 & 8,283 & 17.4593 & up \\
\hline 704 & & $0.0000^{f}$ & SIG & SIG & 68,064 & 212,603 & 899,841 & $2,069,461$ & 13.2205 & down \\
\hline 804 & * & 0.0013 & NS & NS & 8,004 & 5,915 & 148,985 & 250,618 & 18.6134 & down \\
\hline 1004 & * & 0.0023 & NS & NS & 8,004 & 5,915 & 175,554 & 294,631 & 21.9328 & down \\
\hline 1005 & & 0.0012 & SIG & SIG & 83,946 & 197,401 & 354,800 & 440,759 & 4.2265 & down \\
\hline 1109 & & $0.0000^{f}$ & SIG & SIG & 83,319 & 265,430 & 503,759 & $1,143,792$ & 6.0462 & up \\
\hline 1303 & & 0.0012 & SIG & SIG & 364,487 & $1,135,686$ & 155,251 & 282,766 & 2.3477 & up \\
\hline 1309 & * & 0.0043 & NS & NS & 297,936 & 428,232 & 70,605 & 95,878 & 4.2197 & up \\
\hline 1401 & * & 0.0075 & NS & NS & $1,381,518$ & $1,428,751$ & 573,957 & 882,187 & 2.4070 & up \\
\hline 1402 & * & 0.0168 & NS & NS & $1,221,781$ & $1,194,937$ & $2,454,219$ & 2,386,797 & 2.0087 & down \\
\hline 1405 & & 0.0219 & SIG & NS & 536,821 & 589,544 & 164,445 & 364,867 & 3.2644 & up \\
\hline 1704 & & 0.0076 & NS & SIG & 107,476 & 344,505 & 651,980 & $1,109,723$ & 6.0663 & down \\
\hline 2101 & * & 0.0238 & NS & NS & $1,068,400$ & $1,220,098$ & 824,152 & 883,156 & 1.2964 & up \\
\hline 2107 & & 0.0158 & SIG & SIG & 65,274 & 230,953 & 293,355 & 519,761 & 4.4942 & down \\
\hline 2312 & $*$ & 0.0284 & NS & NS & 350,710 & 704,649 & 8,417 & 8,283 & 41.6664 & up \\
\hline 2506 & * & 0.0469 & NS & NS & $1,897,806$ & $1,096,881$ & $2,497,681$ & $1,221,943$ & 1.3161 & down \\
\hline 2507 & * & 0.0012 & NS & NS & 417,660 & 599,615 & $1,048,242$ & $1,050,906$ & 2.5098 & down \\
\hline 2611 & & 0.0498 & SIG & SIG & $1,535,001$ & $1,783,491$ & $2,205,570$ & $2,796,718$ & 1.4369 & down \\
\hline 2614 & & $0.0000^{f}$ & SIG & SIG & 8,004 & 5,915 & 285,838 & 432,421 & 35.7112 & down \\
\hline 2615 & & 0.0008 & SIG & $\mathrm{SIG}$ & 62,288 & 208,601 & 431,929 & $1,018,078$ & 6.9344 & down \\
\hline 2801 & & $0.0000^{f}$ & SIG & SIG & 8,004 & 5,915 & 869,550 & $1,285,546$ & 108.6371 & down \\
\hline 2803 & & 0.0010 & SIG & $\mathrm{SIG}$ & 712,668 & $1,115,376$ & 580,029 & 825,345 & 1.2287 & up \\
\hline 2806 & & 0.0001 & SIG & SIG & 443,822 & $1,084,784$ & 129,571 & 442,770 & 3.4253 & up \\
\hline 3201 & * & 0.0016 & NS & NS & $1,336,983$ & $1,205,431$ & 794,583 & 908,778 & 1.6826 & up \\
\hline
\end{tabular}


Table 3 Significant effects, means, and expression ratio for protein spots with significant beech bark disease (BBD) effect (Continued)

\begin{tabular}{|c|c|c|c|c|c|c|c|c|c|c|}
\hline 3305 & & $0.0000^{f}$ & SIG & SIG & 89,683 & 248,936 & 303,148 & 448,565 & 3.3802 & down \\
\hline 3306 & & 0.0078 & SIG & SIG & 116,998 & 272,340 & 59,537 & 124,166 & 1.9651 & up \\
\hline 3404 & & 0.0082 & SIG & NS & $4,068,995$ & $2,823,334$ & $3,452,364$ & $2,764,490$ & 1.1786 & up \\
\hline 3406 & & $0.0000^{f}$ & SIG & SIG & 44,870 & 125,570 & 462,745 & 547,706 & 10.3130 & down \\
\hline 3412 & & 0.0177 & NS & SIG & 322,035 & 683,636 & 130,942 & 334,190 & 2.4594 & up \\
\hline 3502 & & 0.0188 & SIG & NS & 442,850 & 650,679 & 786,812 & $1,011,948$ & 1.7767 & down \\
\hline 3607 & * & 0.0105 & NS & NS & $2,247,225$ & $2,722,559$ & $1,430,733$ & $2,093,163$ & 1.5707 & up \\
\hline 3702 & * & 0.0001 & NS & NS & 633,076 & $1,243,273$ & $3,792,473$ & $3,730,678$ & 5.9905 & down \\
\hline 3708 & * & 0.0096 & NS & NS & 633,632 & 796,621 & 407,271 & 620,398 & 1.5558 & up \\
\hline 3802 & & 0.0015 & SIG & SIG & 8,004 & 5,915 & 111,820 & 262,030 & 13.9703 & down \\
\hline 3905 & * & 0.0039 & NS & NS & 8,004 & 5,915 & 137,656 & 242,371 & 17.1980 & down \\
\hline 4106 & & 0.0066 & SIG & SIG & 105,844 & 212,452 & 397,149 & 845,289 & 3.7522 & down \\
\hline 4201 & & 0.0010 & NS & SIG & 8,004 & 5,915 & 254,207 & 454,577 & 31.7593 & down \\
\hline 4305 & & $0.0000^{f}$ & SIG & SIG & 46,506 & 128,075 & 470,932 & 768,247 & 10.1263 & down \\
\hline 4706 & * & 0.0481 & NS & NS & $2,739,148$ & $3,047,552$ & 917,692 & $1,062,566$ & 2.9848 & up \\
\hline 4802 & & $0.0000^{f}$ & SIG & SIG & 48,748 & 143,131 & 408,963 & 769,834 & 8.3894 & down \\
\hline 4906 & * & 0.0302 & NS & NS & 873,770 & $1,402,616$ & 628,704 & $1,038,074$ & 1.3898 & up \\
\hline 5301 & & 0.0202 & SIG & SIG & 450,470 & 363,776 & 399,574 & 418,863 & 1.1274 & up \\
\hline 5303 & * & 0.0062 & NS & NS & $3,456,984$ & $1,373,851$ & $5,360,468$ & $2,202,726$ & 1.5506 & down \\
\hline 5505 & * & 0.0009 & NS & NS & 604,226 & 742,627 & $2,522,891$ & $2,358,561$ & 4.1754 & down \\
\hline 5601 & * & 0.0063 & NS & NS & 456,028 & 681,368 & $1,497,166$ & $1,370,793$ & 3.2831 & down \\
\hline 5605 & & $0.0000^{f}$ & SIG & SIG & 124,350 & 177,741 & 8,004 & 5,915 & 15.5356 & up \\
\hline 5606 & & $0.0000^{f}$ & SIG & SIG & 110,019 & 153,695 & 8,004 & 5,915 & 13.7452 & up \\
\hline 5703 & * & 0.0297 & NS & NS & $3,051,574$ & $5,434,263$ & 478,287 & $1,621,642$ & 6.3802 & up \\
\hline 5802 & * & 0.0011 & NS & NS & $1,190,828$ & 915,352 & 508,682 & 528,700 & 2.3410 & up \\
\hline 5908 & & 0.0010 & SIG & SIG & 161,910 & 387,868 & 8,004 & 5,915 & 20.2282 & up \\
\hline 5909 & & 0.0012 & SIG & SIG & 8,417 & 8,283 & 143,259 & 352,729 & 17.0200 & down \\
\hline 6208 & & 0.0297 & SIG & SIG & 186,317 & 276,277 & 80,227 & 222,514 & 2.3224 & up \\
\hline 6303 & & 0.0328 & NS & SIG & 253,951 & 331,973 & 397,930 & 349,663 & 1.5670 & down \\
\hline 6304 & & 0.0018 & NS & SIG & $2,888,828$ & $3,401,904$ & 820,317 & $1,445,597$ & 3.5216 & up \\
\hline 6306 & * & 0.0099 & NS & NS & $1,800,296$ & $1,365,419$ & $2,730,178$ & $2,859,788$ & 1.5165 & down \\
\hline 6501 & * & 0.0318 & NS & NS & $1,468,936$ & $1,183,570$ & 604,825 & $1,255,327$ & 2.4287 & up \\
\hline 6707 & $*$ & 0.0193 & NS & NS & $11,970,218$ & $12,515,695$ & $6,247,307$ & $6,508,633$ & 1.9161 & up \\
\hline 6709 & * & 0.0238 & NS & NS & $8,974,280$ & $5,564,143$ & $5,289,975$ & $6,857,853$ & 1.6965 & up \\
\hline 6710 & $*$ & 0.0238 & NS & NS & $1,400,824$ & $2,287,877$ & 404,306 & 569,323 & 3.4648 & up \\
\hline 6807 & & 0.0070 & SIG & SIG & 118,758 & 291,241 & 8,004 & 5,915 & 14.8370 & up \\
\hline 6808 & & 0.0009 & SIG & SIG & $1,174,714$ & $1,224,320$ & 239,503 & 580,829 & 4.9048 & up \\
\hline 6809 & & 0.0002 & SIG & SIG & 376,863 & 587,605 & 95,365 & 213,349 & 3.9518 & up \\
\hline 7009 & & 0.0004 & SIG & SIG & 175,933 & 391,166 & 265,335 & 410,728 & 1.5082 & down \\
\hline 7106 & & $0.0000^{f}$ & SIG & SIG & 962,980 & $1,282,010$ & 39,820 & 101,833 & 24.1835 & up \\
\hline 7107 & * & 0.0009 & NS & NS & 387,387 & 501,608 & 50,505 & 130,300 & 7.6703 & up \\
\hline 7110 & * & 0.0245 & NS & NS & 84,648 & 139,984 & 256,705 & 307,196 & 3.0326 & down \\
\hline 7111 & & $0.0000^{f}$ & SIG & SIG & 658,929 & $1,249,222$ & 148,370 & 341,871 & 4.4411 & up \\
\hline 7112 & & 0.0022 & NS & $S I G$ & 73,598 & 133,128 & 8,004 & 5,915 & 9.1949 & up \\
\hline
\end{tabular}


Table 3 Significant effects, means, and expression ratio for protein spots with significant beech bark disease (BBD) effect (Continued)

\begin{tabular}{|c|c|c|c|c|c|c|c|c|c|c|}
\hline 7203 & & 0.0261 & SIG & NS & $1,311,783$ & 732,419 & 927,380 & 799,253 & 1.4145 & up \\
\hline 7207 & & 0.0186 & SIG & NS & 60,957 & 126,879 & 21,092 & 40,907 & 2.8901 & up \\
\hline 7303 & & 0.0052 & SIG & SIG & 246,921 & 602,207 & 615,797 & $1,105,070$ & 2.4939 & down \\
\hline 7408 & * & 0.0126 & NS & NS & $3,049,802$ & $2,337,843$ & $1,336,825$ & $1,182,165$ & 2.2814 & up \\
\hline 7510 & & 0.0098 & SIG & SIG & 305,493 & 558,423 & 397,578 & $1,073,627$ & 1.3014 & down \\
\hline 7602 & * & 0.0412 & NS & NS & 7,296,723 & $7,488,294$ & $3,609,984$ & $5,505,222$ & 2.0213 & up \\
\hline 7609 & & 0.0016 & SIG & SIG & 381,570 & 946,188 & 8,004 & 5,915 & 47.6713 & up \\
\hline 7803 & * & 0.0238 & NS & NS & 716,697 & 474,399 & 341,082 & 391,607 & 2.1012 & up \\
\hline 7808 & $*$ & 0.0075 & NS & NS & 704,023 & 626,350 & 305,591 & 487,526 & 2.3038 & up \\
\hline 7809 & * & 0.0267 & NS & NS & 582,174 & $1,475,329$ & 8,004 & 5,915 & 72.7338 & up \\
\hline 7810 & $*$ & 0.0297 & NS & NS & 442,246 & $1,119,936$ & 8,004 & 5,915 & 55.2519 & up \\
\hline 8002 & * & 0.0082 & NS & NS & 175,231 & 300,741 & 22,986 & 74,010 & 7.6235 & up \\
\hline 8104 & * & 0.0188 & NS & NS & $1,096,503$ & 874,012 & 394,837 & 541,448 & 2.7771 & up \\
\hline 8202 & & 0.0230 & SIG & NS & 29,407 & 58,674 & 56,069 & 97,123 & 1.9066 & down \\
\hline 8303 & * & 0.0311 & NS & NS & $1,850,424$ & $1,058,481$ & $1,293,017$ & $1,164,851$ & 1.4311 & up \\
\hline 8309 & * & 0.0008 & NS & NS & 340,383 & 449,968 & 902,563 & 716,532 & 2.6516 & down \\
\hline 8311 & & 0.0318 & SIG & NS & 85,387 & 133,171 & 26,149 & 58,170 & 3.2654 & up \\
\hline 8312 & $*$ & 0.0498 & NS & NS & 306,017 & 504,921 & 549,086 & 736,070 & 1.7943 & down \\
\hline 8503 & * & 0.0193 & NS & NS & 587,330 & 907,526 & 123,323 & 558,974 & 4.7626 & up \\
\hline 8506 & * & 0.0010 & NS & NS & $1,709,206$ & $2,026,022$ & 334,292 & 701,018 & 5.1129 & up \\
\hline 8508 & * & 0.0158 & NS & NS & $1,395,005$ & $1,198,636$ & 633,833 & 820,628 & 2.2009 & up \\
\hline 8512 & $*$ & 0.0012 & SIG & NS & 8,417 & 8,283 & $1,173,776$ & $1,657,190$ & 139.4515 & down \\
\hline 8604 & & 0.0267 & SIG & SIG & $2,262,933$ & $3,169,913$ & $1,147,023$ & $2,200,353$ & 1.9729 & up \\
\hline 8607 & & 0.0020 & SIG & NS & $1,865,790$ & $3,095,124$ & 369,996 & 861,448 & 5.0427 & up \\
\hline 8708 & * & 0.0076 & NS & NS & 177,743 & 461,377 & 632,706 & $1,403,084$ & 3.5597 & down \\
\hline 8801 & * & 0.0316 & NS & NS & 751,522 & 573,471 & 517,615 & 499,829 & 1.4519 & up \\
\hline 8802 & * & 0.0034 & NS & NS & 511,552 & 398,150 & 269,962 & 349,834 & 1.8949 & up \\
\hline 9302 & * & 0.0238 & NS & NS & 710,525 & 848,447 & $1,268,492$ & $1,442,080$ & 1.7853 & down \\
\hline 9305 & & 0.0085 & SIG & SIG & 301,789 & 462,883 & 368,200 & 614,918 & 1.2201 & down \\
\hline 9401 & & 0.0192 & SIG & NS & 717,956 & 641,915 & 419,853 & 410,107 & 1.7100 & up \\
\hline 9402 & & 0.0096 & SIG & SIG & 63,499 & 152,855 & 120,990 & 362,645 & 1.9054 & down \\
\hline 9802 & & 0.0021 & SIG & SIG & 112,023 & 263,531 & 8,004 & 5,915 & 13.9956 & up \\
\hline
\end{tabular}

An asterisk to the right of the spot number indicates a spot with significant BBD effect and non-significant STAND and STANDxBBD interaction effect, and are the primary targets for biomarker development.

BBD effect with q-value $<0.05$. q-value controls the False Discovery rate, and $q<0.05$ allows for no more than 26 false positives in the 425 spots tested.

bSTAND effect with $\mathrm{p}<0.0001$ (Bonferroni adjusted critical value).

'STANDXBBD interaction effect with $\mathrm{p}<0.0001$ (Bonferroni adjusted critical value).

dGaussian image spot intensity after normalization expressed in counts. Mean is the mean over the three technical replicates per tree for all trees in that disease condition. SD is standard deviation.

e Ratio is ratio of larger mean over smaller mean.

Mean = Gaussian image spot intensity after normalization expressed in counts. Mean is the mean over the three technical replicates for all trees in that disease condition. $\mathrm{SD}=$ standard deviation.

${ }^{f} Q$-values displayed as 0.0000 are positive values $<0.0001$. See additional table 1 for actual q-values.

chronic disease, with significant associated bark damage including cracking, callous formation [1], and likely secondary local stress effects such as dehydration or nutrient and photosynthate transport disruptions. These bark stress factors may induce other, poorly understood sets of stress responses. The signalling pathways triggered by these multiple sources of stress are also likely interconnected through their regulatory pathways, which in other plant systems have been shown to be antagonistic, further adding to the complexity and variation of the 
Table 4 Protein spots analyzed by MS/MS

\begin{tabular}{|c|c|c|c|c|c|c|c|c|c|c|}
\hline Spotnumber & $\begin{array}{l}\text { Tree \& core } \\
\text { number }\end{array}$ & 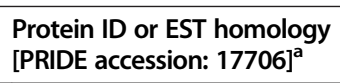 & $\begin{array}{l}\text { NCBI or EST Accession } \\
\text { Number }\end{array}$ & MascotScore $^{c}$ & $\begin{array}{l}\text { Percent } \\
\text { Coverage }\end{array}$ & $\begin{array}{l}\text { Matching } \\
\text { peptides }\end{array}$ & Peptide sequence $^{d}$ & $\begin{array}{l}\text { Peptide } \\
\text { score }^{\mathrm{e}}\end{array}$ & $\mathrm{m} / \mathrm{z}$ & $\operatorname{Mr}(\operatorname{expt})$ \\
\hline \multirow[t]{4}{*}{$109 * f$} & 1504_B6 & polyphenol oxidase & ABWP2_088861_1753_2628 & 180 & 33 & 2 & TEFAGSFVNVPSK & 72.2 & 692.1743 & 1382.334 \\
\hline & & & & & & 4 & GVATIGGIK & 47.2 & 408.4638 & 814.913 \\
\hline & & & & & & 2 & GVATIGGIKIQFVS & 233.8 & 695.8282 & 1389.6418 \\
\hline & & & & & & 2 & IQFVS & 26.9 & 593.343 & 592.3357 \\
\hline \multirow[t]{5}{*}{307} & 1504_H5 & unnamed protein product & gi|157327346 & 735 & 44 & 2 & NILFVISKPDVFK & 71.3 & 760.6617 & 1519.3088 \\
\hline & & & & & & 4 & SPTSDTYIFGEAK & 73.9 & 758.1431 & 1514.2716 \\
\hline & & & & & & 6 & IEDLSSQLQTQAAEQFK & 107.3 & 969.241 & 1936.4674 \\
\hline & & & & & & 2 & DIELVṂTQAGVSR & 75.7 & 718.1302 & 1434.2458 \\
\hline & & & & & & 4 & DIELVMTQAGVSR & 98.1 & 709.7012 & 1417.3878 \\
\hline \multirow[t]{3}{*}{307} & 1504_H5 & alpha nascent polypeptide & CCWP2_066819_3631_1428 & 255 & 52 & 2 & NILFVISKPDVFK & 71.3 & 760.6617 & 1519.3088 \\
\hline & & associated complex & & & & 4 & SPTSDTYIFGEAK & 74.8 & 758.1431 & 1514.2716 \\
\hline & & & & & & 5 & IEDLSSQLQTQAAEQFK & 108.5 & 969.241 & 1936.4674 \\
\hline 505 & 1504_A6 & none & none & & & & & & & \\
\hline \multirow[t]{2}{*}{$1004^{*}$} & CM02d_C1 & thioredoxin & WOA_067025_0312_1563 & 120 & 25 & 2 & VNTDESSSIATR & 76.5 & 640.5275 & 1279.0404 \\
\hline & & & & & & 2 & STLTTSIEK & 46.2 & 490.4947 & 978.9748 \\
\hline \multirow[t]{2}{*}{$2101^{*}$} & 1504_G7 & ribonuclease activity regulator & CCNHS_110619_3244_2001 & 214 & 24 & 2 & VFEDNVLVR & 57.1 & 546.048 & 1090.0814 \\
\hline & & & & & & 2 & VLWDGGGSLR & 67.6 & 537.0695 & 1072.1244 \\
\hline \multirow[t]{3}{*}{$2101^{*}$} & 1504_G7 & dimethylmenaquinone & gi|15232963 & 199 & 21 & 4 & ALQPVFQIYGR & 46.8 & 647.1753 & 1292.336 \\
\hline & & $\begin{array}{l}\text { methyltransferase family } \\
\text { protein }\end{array}$ & & & & 2 & VLWDGGGSLR & 78.9 & 537.0695 & 1072.1244 \\
\hline & & & & & & 2 & DVDEINGCDIGVR & 66.5 & 731.6575 & 1461.3004 \\
\hline \multirow[t]{2}{*}{$2101^{*}$} & CM02d_C3 & $\begin{array}{l}\text { Eukaryotic translation } \\
\text { initiation }\end{array}$ & gi|3024017 & 75 & 19 & 1 & ELVFKEDGQEYAQVLR & 45.4 & 642.4005 & 1924.1797 \\
\hline & & factor $1 \mathrm{~A}$ & & & & 2 & DYQDDKADVILK & 52.4 & 712.098 & 1422.1814 \\
\hline \multirow[t]{3}{*}{3101} & CM02d_E5 & heat shock protein & ROA_067935_0424_3607 & 120 & 22 & 2 & ADLPGLK & 39.2 & 357.4087 & 712.8028 \\
\hline & & [homologous match] & & & & 1 & KEEVKVEVEEGR & 32.3 & 477.7563 & 1430.2471 \\
\hline & & & & & & 2 & EEVKVEVEEGR & 53.2 & 652.0981 & 1302.1816 \\
\hline \multirow[t]{5}{*}{4103} & 1504_F5 & 17.7 kDa heat shock protein & gi|1235898 & 238 & 26 & 2 & ADLPGLK & 39.7 & 357.3622 & 712.7098 \\
\hline & & & & & & 7 & KEEVKVEVEEGR & 79.9 & 715.8541 & 1429.6936 \\
\hline & & & & & & 5 & EEVKVEVEEGR & 58.7 & 652.0201 & 1302.0256 \\
\hline & & & & & & 5 & VLQISGER & 30.3 & 451.5257 & 901.0368 \\
\hline & & & & & & 5 & AAMENGVLTVTVPK & 32.4 & 723.6349 & 1445.2552 \\
\hline
\end{tabular}


Table 4 Protein spots analyzed by MS/MS (Continued)

\begin{tabular}{|c|c|c|c|c|c|c|c|c|c|c|}
\hline \multirow[t]{11}{*}{5001} & CM02d_F5 & $\begin{array}{l}\text { stress and pathogenesis- } \\
\text { related }\end{array}$ & gi|3901018 & 858 & 65 & 3 & GVFTYESENTSVIPPAR & 84.8 & 934.2761 & 1866.5376 \\
\hline & & protein & & & & 13 & AFVLDADNLIPK & 30.5 & 659.2544 & 1316.4942 \\
\hline & & & & & & 2 & AFVLDADNLIPKVAPQSIK & 42.0 & 680.6581 & 2038.9525 \\
\hline & & & & & & 7 & STETLEGDGGPGTIK & 44.6 & 731.3245 & 1460.6344 \\
\hline & & & & & & 5 & STETLEGDGGPGTIKK & 66.3 & 795.6741 & 1589.3336 \\
\hline & & & & & & 3 & KITFGEGSQFK & 60.5 & 414.6845 & 1241.0317 \\
\hline & & & & & & 4 & ITFGEGSQFK & 49.7 & 1113.4546 & 1112.4473 \\
\hline & & & & & & 1 & ISYEIKIVASPDGGSLLK & 25.6 & 630.7996 & 1889.377 \\
\hline & & & & & & 6 & IVASPDGGSLLK & 63.3 & 578.6285 & 1155.2424 \\
\hline & & & & & & 2 & GNHEIKEEEVK & 55.8 & 656.6091 & 1311.2036 \\
\hline & & & & & & 10 & AVEAYLLAHPDAYN & 47.7 & 774.1621 & 1546.3096 \\
\hline \multirow[t]{5}{*}{5002} & 1504_G5 & superoxide dismutase & gi|38228697 & 263 & 40 & 1 & HAGDLGNVNVGDDGTVSFTIIDK & 72.8 & 782.4584 & 2344.3534 \\
\hline & & & & & & 4 & QIPLCGPNSIIIGR & 53.1 & 713.6349 & 1425.2552 \\
\hline & & & & & & 4 & AWHHGPDDLGK & 55.5 & 661.611 & 1321.2074 \\
\hline & & & & & & 2 & STGNAGGRIACGIIGLQG & 45.4 & 851.7599 & 1701.5052 \\
\hline & & & & & & 4 & IACGI|IGLG & 45.6 & 1001.4746 & 1000.4673 \\
\hline \multirow[t]{3}{*}{$5303^{*}$} & 1504_F7 & triosephosphate isomerase & ABWP2_034459_1554_1107 & 250 & 52 & 3 & VASPAQAQEVHFGLR & 66.9 & 805.7179 & 1609.4212 \\
\hline & & & & & & 3 & KWLQVNTSPEVAATTR & 48.9 & 601.354 & 1801.0402 \\
\hline & & & & & & 1 & ॥YGGSVNGANCK & 63.6 & 677.621 & 1353.2274 \\
\hline \multirow[t]{5}{*}{$7107^{*}$} & 1504_B7 & $\begin{array}{l}\text { unknown, CBS domain } \\
\text { protein }\end{array}$ & CCWP1_110480_2642_0865 & 274 & 53 & 2 & VGDIMTTEENK & 49.3 & 576.5307 & 1151.0468 \\
\hline & & & & & & 2 & VGDIMTEEENKLITVTLDTK & 59.3 & 713.168 & 2136.4822 \\
\hline & & & & & & 1 & LITVTLDTK & 45.3 & 502.5542 & 1003.0938 \\
\hline & & & & & & 2 & GMIGMVISIGDWR & 84.6 & 683.4301 & 1364.8456 \\
\hline & & & & & & 2 & LNAFIQGGY & 38.5 & 982.3546 & 981.3473 \\
\hline \multirow[t]{4}{*}{$7110^{*}$} & 1504_C7 & $\begin{array}{l}\text { unknown, CBS domain } \\
\text { protein }\end{array}$ & CCWP1_110480_2642_0865 & 230 & 53 & 2 & VGDIMTEENK & 56.2 & 576.5154 & 1151.0162 \\
\hline & & & & & & 2 & VGDIMTEEENKLITVTLDTK & 61.3 & 713.1682 & 2136.4828 \\
\hline & & & & & & 2 & GMIGMVSIGDWR & 80.2 & 683.6461 & 1365.2776 \\
\hline & & & & & & 2 & LNAFIQGGY & 40.0 & 982.4321 & 981.4248 \\
\hline
\end{tabular}


Table 4 Protein spots analyzed by MS/MS (Continued)

\begin{tabular}{|c|c|c|c|c|c|c|c|c|c|c|}
\hline \multirow[t]{4}{*}{$7408^{*}$} & CM02d_A4 & annexin & ACHS1n_159819_2640_1758 & 209 & 66 & 2 & WTSSNQVLMEIACTR & 84.0 & 906.6581 & 1811.3016 \\
\hline & & & & & & 2 & SSHDLLLAR & 37.8 & 506.6234 & 1011.2322 \\
\hline & & & & & & 1 & SLEEDVAYHTTGDFR & 28.8 & 580.6663 & 1738.9771 \\
\hline & & & & & & 2 & YEGDEVNMTLAK & 60.4 & 693.4765 & 1384.9384 \\
\hline \multirow[t]{4}{*}{$7408^{*}$} & CM02d_A4 & isoflavone reductase like & ABWP1_026351_0296_1851 & 203 & 37 & 1 & SKILIIGGTGYIGK & 57.7 & 710.562 & 1419.1094 \\
\hline & & & & & & 2 & ILIIGGTGY|GK & 70.4 & 602.8907 & 1203.7668 \\
\hline & & & & & & 2 & SGHPTFALVR & 36.0 & 542.7687 & 1083.5228 \\
\hline & & & & & & 2 & ESTVSDPVK & 38.7 & 481.347 & 960.6794 \\
\hline \multirow[t]{6}{*}{$7602^{*}$} & CM02d_B4 & dehydrin & ABWP1_045441_0451_3051 & 225 & 53 & 1 & VFEKIPGAGNK & 46.8 & 580.3038 & 1158.593 \\
\hline & & [homologous match] & & & & 1 & IPGAGNKDR & 40.9 & 465.1174 & 928.2202 \\
\hline & & & & & & 2 & DRVQGEQYR & 41.0 & 576.0114 & 1150.0082 \\
\hline & & & & & & 1 & GAMDKVFEK & 30.4 & 521.1149 & 1040.2152 \\
\hline & & & & & & 2 & IPGAGNKDK & 43.2 & 450.2458 & 898.477 \\
\hline & & & & & & 2 & DKVQGDQYR & 34.7 & 555.0321 & 1108.0496 \\
\hline 8002 & CM02d_E4 & none & none & & & & & & & \\
\hline \multirow[t]{2}{*}{$8104^{*}$} & 1504_C9 & $\begin{array}{l}\text { glycine-rich RNA binding } \\
\text { protein }\end{array}$ & ABWP1_015278_0178_1196 & 100 & 24 & 2 & AFSPYGEILESK & 49.6 & 671.1248 & 1340.235 \\
\hline & & [homologous match] & & & & 2 & NITVNEAQSR & 50.5 & 566.5034 & 1130.9922 \\
\hline \multirow[t]{2}{*}{$8104^{*}$} & CM02d_F4 & $\begin{array}{l}\text { glycine-rich RNA-binding } \\
\text { protein }\end{array}$ & ABWP1_015278_0178_1196 & 97 & 25 & 2 & AFSPYGEILESK & 50.4 & 671.1497 & 1340.2848 \\
\hline & & [homologous match] & & & & 2 & GFGFVTFSNEK & 46.6 & 617.2156 & 1232.4166 \\
\hline 8202 & 1504_D9 & none & none & & & & & & & \\
\hline 8303 & 1504_H8 & none & none & & & & & & & \\
\hline 8309 & 1504_A9 & none & none & & & & & & & \\
\hline 8309 & CM02d_A2 & none & none & & & & & & & \\
\hline \multirow[t]{3}{*}{$8506^{*}$} & 1504_F8 & glyceraldehyde 3-phosphate & ROB_045015_1217_3381 & 176 & 57 & 2 & AASFNIIPSSTGAAK & 39.8 & 718.1123 & 1434.21 \\
\hline & & dehydrogenase, cytosolic & & & & 2 & VPTVDVSWDLTVR & 82.6 & 750.6729 & 1499.3312 \\
\hline & & & & & & 2 & FGIVEGLMTTVHSITATQK & 54.0 & 684.0022 & 2048.9848 \\
\hline \multirow[t]{5}{*}{8607} & 1504_E8 & glyceraldehyde-3-phosphate & gi|120666 & 237 & 21 & 2 & FGIVEGLMTTVHSITATQK & 38.7 & 684.0699 & 2049.1879 \\
\hline & & dehydrogenase, cytosolic & & & & 2 & AASFNIIPSSTGAAK & 47.5 & 718.1649 & 1434.3152 \\
\hline & & & & & & 2 & VPTVDVSWDLTVR & 88.8 & 750.368 & 1498.7214 \\
\hline & & & & & & 4 & AGIALNDNFVK & 37.9 & 581.4758 & 1160.937 \\
\hline & & & & & & 2 & LVSWYDNEWGYSTR & 72.0 & 888.5875 & 1775.1604 \\
\hline
\end{tabular}


Table 4 Protein spots analyzed by MS/MS (Continued)

\begin{tabular}{|c|c|c|c|c|c|c|c|c|c|c|}
\hline \multirow[t]{2}{*}{8607} & CM02d_C2 & Ran binding protein & ABWP1_041027_0417_3079 & 146 & 33 & 4 & LEEVAVITGEEDETSILDLK & 117.4 & 1096.213 & 2190.4114 \\
\hline & & & & & & 2 & FDKDGNQWK & 28.9 & 569.5167 & 1137.0188 \\
\hline \multirow[t]{4}{*}{$9302^{*}$} & 1504_G8 & $\begin{array}{l}\text { germin-like/unknown/ } \\
\text { predicted }\end{array}$ & CCNHS_162322_3610_0519 & 282 & 48 & 3 & IPGLNTLGVSLSR & 85.5 & 663.9008 & 1325.787 \\
\hline & & & & & & 3 & IDYAPGGLNPPHTHPR & 77.0 & 872.1409 & 1742.2672 \\
\hline & & & & & & 2 & SIKKGEIFVFPK & 66.0 & 697.1452 & 1392.2758 \\
\hline & & & & & & 4 & KGEIFVFPK & 53.6 & 532.7322 & 1063.4498 \\
\hline
\end{tabular}

9401 CM02d_B5 none

aldentification of the NCBI protein match, or for EST peptide matches, the BLASTP identification of the EST. Mass spectrometry data and identifications have been submitted to the PRIDE database. See the field 'gel spot identifier' in

the Identification Detail View to review individual spot spectra and identifications.
${ }^{b}$ NCBI number of the matching protein, or the Fagaceae genomics EST ID for peptided matching EST's.

'MASCOT score is a probability based score. Reported scores are sums of peptide identifications that are significant (except homologous where noted), and higher scores are equivalent to lower probability of an equal or better

match occurring at random.
mased score. Reported scores are sums of peptide identifications that are significant (except homologous where noted), and higher scores are equivalent to lower probability of an equal or better match occurring at random.

Underline indicates an oxidized residue. Bold indicates a missed trypsin cleavage.

$f_{*}^{*}$ indicates spot with significant BBD effect and non-significant STAND and STANDXBBD interaction effect, and are the primary targets for biomarker development. 
Table 5 Previously published stress response of proteins identified by MS/MS

\begin{tabular}{|c|c|c|c|c|c|}
\hline Category & Protein (or protein family) & Spot(s) & $\begin{array}{l}\text { Quantification in } \\
\text { healthy trees relative } \\
\text { to diseased trees }\end{array}$ & $\begin{array}{l}\text { Previously published stress } \\
\text { response }\end{array}$ & References \\
\hline \multirow[t]{4}{*}{ reactive oxygen species } & polyphenol oxidase & 109 & up & wound, pathogen, insect & [17-19] \\
\hline & $\begin{array}{l}\text { glyceraldehyde-3-phosphate } \\
\text { dehydrogenase }\end{array}$ & $85068607,1^{\text {st }}$ ID & up & $\begin{array}{l}\text { hypersensitive response mimic, } \\
\text { hydrogen peroxide, wounding }\end{array}$ & [20-23] \\
\hline & annexin & $7408,1^{\text {st }}$ ID & up & oxidative stress & [24-26] \\
\hline & thioredoxin & 1004 & down & oxidative stress & {$[27-30]$} \\
\hline \multirow[t]{3}{*}{$\begin{array}{l}\text { stress and pathogenesis } \\
\text { related proteins }\end{array}$} & dehydrins & 7602 & up & $\begin{array}{l}\text { drought, low temperature, freezing } \\
\text { salinity, abscisic acid treatment }\end{array}$ & [31-33] \\
\hline & isoflavone reductase-like protein & $7408,2^{\text {nd }}$ ID & up & lignification & [34] \\
\hline & germin-like proteins & 9302 & down & pathogen, aluminum, wounding & [35-39] \\
\hline \multirow[t]{4}{*}{$\begin{array}{l}\text { transcription and } \\
\text { translation }\end{array}$} & $\begin{array}{l}\text { eukayrotic translation initiation } \\
\text { factor (eif1) }\end{array}$ & 2101 & up & salt & {$[40,41]$} \\
\hline & glycine-rich RNA binding protein & 8104 & up & $\begin{array}{l}\text { hydrogen peroxide, flooding, cold, } \\
\text { drought, salinity, abscisic acid } \\
\text { treatment }\end{array}$ & {$[42,43]$} \\
\hline & $\begin{array}{l}\text { alpha nascent polypeptide } \\
\text { associated complex } 1 \text { (aNAC1) }\end{array}$ & 307 & up & $\begin{array}{l}\text { salt, systemic acquired resistance } \\
\text { inducer }\end{array}$ & {$[44-46]$} \\
\hline & Ran binding protein(RanBP) & $8607,2^{\text {nd }} \mathrm{ID}$ & & auxin treatment & {$[47]$} \\
\hline \multirow[t]{2}{*}{$\begin{array}{l}\text { unknown, or previously } \\
\text { metabolic only }\end{array}$} & triose-phosphate isomerase (TPI) & 5303 & down & $\begin{array}{l}\text { systemic acquired resistance inducer, } \\
\text { abscisic acid treatment, pathogen }\end{array}$ & {$[46-49]$} \\
\hline & CBS domain containing protein & 7107,7110 & up, down & stress responsive & [50] \\
\hline
\end{tabular}

Many of the proteins with a BBD effect that were identified by MS/MS are previously published as stress responsive in other plant systems.

response of the tree to the causal components of beech bark disease [55].

A high level of genetic diversity across geographical regions was previously reported in maritime pine [54]. In our study, genetic diversity across geographical regions is combined with the complexity of host responses to a dual pathogen disease system making the high number of spots identified in samples (up to 522 per tree, total of 987) not unexpected. Resolution of similar ranges of spot numbers (from 451 up to 753) have been previously reported in other plant species using similar supplies, equipment, and technical approaches [56-60]. Technical effects were significant for only six spots indicating that the observed differences between gels and samples were accounted for by the experimental design and analysis. Bark samples were anecdotally different for some visible features (such as chlorophyll content) that may vary by season, micro-site, or sample. Several spots that were not identified by tandem mass spectrometry had some (non-significant) homology to Fagaceae EST's, suggesting matching of unidentified peptides may be possible as additional woody plant, bark, and particularly American beech sequences become available. The low number of identified proteins in forest tree proteomic studies in general is a direct reflection of the lack of genomic DNA, EST and protein sequence data entries in public databases [51] for forest trees and for woody tissues.

The genetic complexity of the sample unit, the sampling across a wide geographic area, and the complexity of the BBD phenotype all contribute to possible protein differences between trees in the study. This complexity, especially combined with typical technical sources of variation, required careful study design and more elaborate statistical considerations than many proteomics studies. Identification of up to 101 protein spots unique to an individual tree emphasizes the genetic diversity captured in our study. One-hundred-twenty protein spots (22.5\% of the matched spots) were identified as BBD significant despite the experimental complexity, so the experiment was effective at finding proteins of interest. We controlled the false discovery rate to $5 \%$ (using the q-value), so we would expect only six of the 120 proteins identified as differentially expressed to be erroneous.

\section{Sequenced spots have homology to known stress, insect,} and pathogen related proteins in other plants

Most of the proteins identified by MS/MS in this study have homology to proteins known to be involved in stress responses in other plants (Table 5). The variety of biological responses to which these proteins are linked are consistent with the complexity of BBD. Since $\mathrm{BBD}$ has both an insect and a fungal component, it is not unexpected that both insect and pathogen related proteins would be found. BBD is also a long-term disease with both bark damage and significant healing as part of the physiology of the disease. Wounding, reactive oxygen species and drought responsive proteins are 


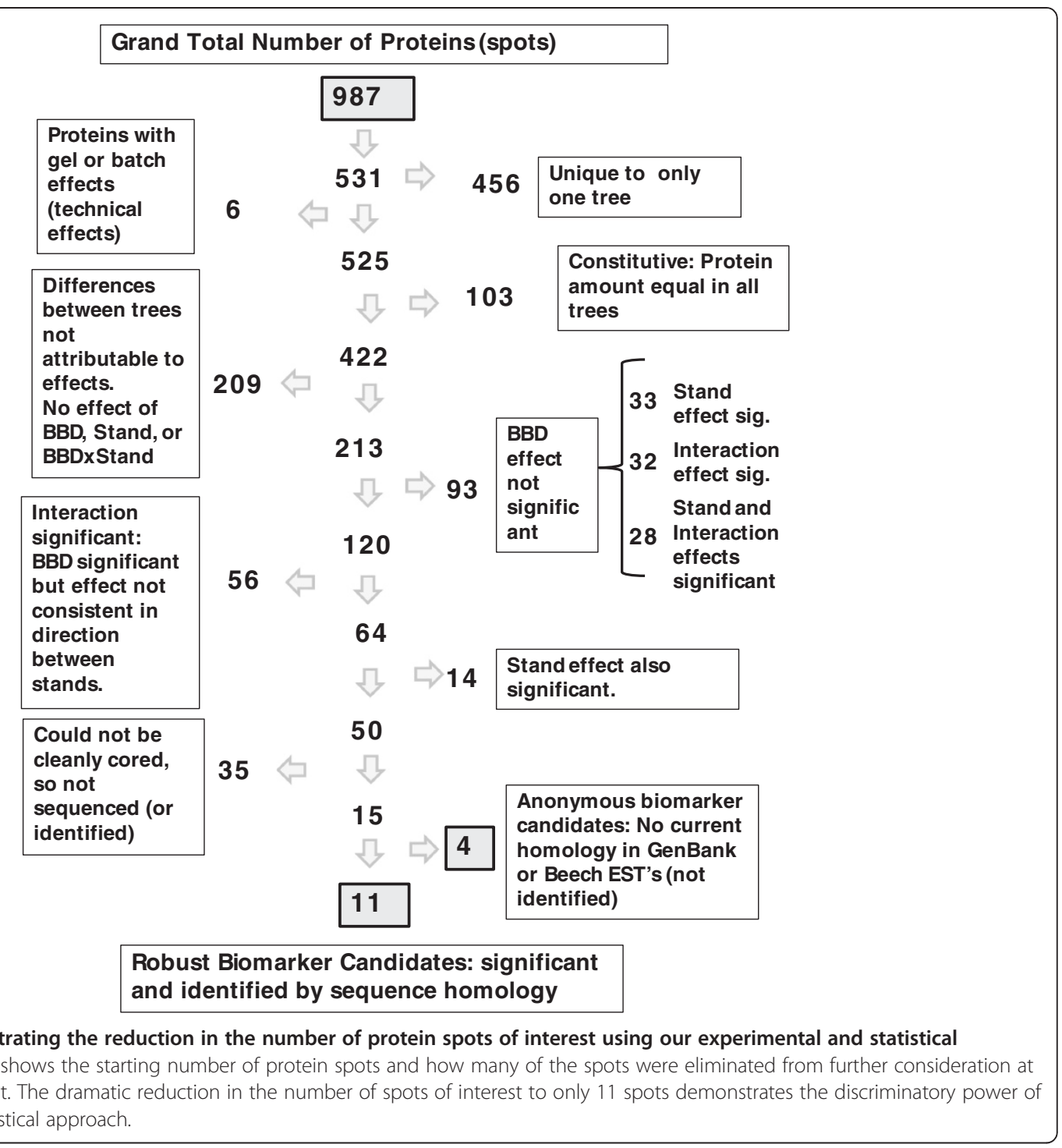

also expected. The variety of stress responses apparently influenced by BBD along with the identification of proteins involved both in transcription and translation control and basic metabolic responses, supports a model that beech trees have an active physiological response to BBD. These proteins are good targets for further research to understand the pathways involved in response to BBD.

The proteins identified in this study also expand on recent studies of the proteome of European Beech (Fagus sylvatica L.) [61-63]. While our study is the first publication of the proteome of bark tissue or any tissue from mature trees from Fagus, a few proteins identified in our study were also identified in other studies in Fagus. Of particular interest, Valcu et al. [63] identified triosephosphate isomerase (TPI) as having a lower protein level in wounded leaves, while we found the protein to have higher expression in the bark of BBD diseased trees. Beech trees afflicted with beech bark disease would be responding to both scale feeding and pathogen infection (Neonectria spp.). The higher expression of TPI in diseased trees is consistent with the reports of higher expression of TPI in response to a fungal pathogen in Brassica carinata [48]. Further study of TPI to understand its role in defense in different tissues and different stages of beech bark disease infestation is certainly warranted.

\section{Biomarker candidates for further analysis}

The long term goal of our research program is to identify broadly useful markers for BBD resistance that forest managers can use to plan for and mitigate $\mathrm{BBD}$ damage as it spreads to new regions and stands. A resistance biomarker could also be used to expedite the selection and breeding of scale-resistant trees in on-going tree improvement programs [10]. 
Twelve of the sequence identified spots (marked with an asterisk in both Table 3 and Table 4) showed a significant BBD effect and no effect of stand or BBDxStand interaction effect. Proteins different in healthy versus diseased trees regardless of stand (i.e. across stands) are the best biomarker candidates. These proteins are the most likely to be linked to resistance or susceptibility across broad geographic and genetic ranges, and so are the highest priority for follow-up study and biomarker development. Quantitation of these proteins in additional trees, especially in trees from other stands and regions, will be important in determining if these proteins can be used as biomarkers. Additional 2-DE gel studies, or development of antibody based (western blot, or ELISA) methods will facilitate this. RNA expression studies may also be helpful in understanding which proteins (genes) are the best markers for BBD resistant trees. An additional five spots that weren't identified by sequence homology fit into the same class and may be identified in the future as additional sequence becomes available. Often spots unidentified by MS/MS are dropped from further study. But given both the low amount of Fagus sequence available for comparison and the low amount of sequences in the database from bark tissues and forest trees in general, these spots are still good candidates for further study for biomarker development.

Proteins for which BBD effect is significant along with stand, interaction, or both require more careful interpretation, but may still become useful biomarkers. In combination with significant stand and BBD effects, a significant interaction effect means that the direction of association of a BBD effect (e.g. increased protein in healthy trees) is not consistent in the different stands. When STAND and BBD effects are both significant (but with a non-significant interaction effect), the protein may not be consistently distributed in American beech and may be present in only some of the stands managers wish to screen. Both of these groups of proteins are less attractive as biomarker candidates, but interpretation of them along with other higher priority candidate proteins may be insightful.

Most of the proteins identified by sequence have been found to be responsive to stress, insect, or pathogens in other plant systems, and are differentially expressed between the healthy and diseased trees. A small number of these proteins will be selected for further study, with priority given to those that are higher in the healthy trees and are predicted to be involved in insect resistance because of the known scale-insect resistance of the healthy trees and the requirement of scale feeding induced bark wounding to provide subsequent entryway for Neonectria infection $[1,2,7]$. Polyphenol oxidase and glyceraldehyde dehydrogenase both fall into this class and are considered high priority for further analysis. It will be important to continue validation of expression results using additional trees from the same stands, and additional stands from new geographic regions. In addition, the best biomarker protein may not be the one identified in this study, but rather a protein acting upstream in a response pathway, or regulating a response pathway(s). Further characterization of the biochemical pathways, and their induction through time, season, and spatially through the tree will be important. It is possible the proteins identified in this study will coincide with quantitative trait loci for scale resistance.

\section{Conclusions}

American beech is an ecologically important species in many North American forests, only a portion of which are currently impacted by BBD [64]. Development of management options to reduce the economic losses and ecological costs of BBD are critically needed. This study has identified protein spots differentially expressed in the bark of healthy, scale-resistant trees and BBD-susceptible trees. This identification suggests that American beech has an active physiological response to BBD. Confirming this response is an important first step in understanding how BBD may progress physiologically and mechanistically in BBD susceptible trees, and how BBD resistance may be manifested. Additionally the results of this study should support and complement on-going strategies to find biomarkers for $\mathrm{BBD}$ resistance.

\section{Methods}

\section{Selection of beech trees and collection of bark samples}

Ten healthy trees were identified in seven stands in Fredericton, New Brunswick, Canada (Table 1). Healthy trees comprised only $5 \%$ of the beech trees in this area and were included in this study only if they were greater than $15 \mathrm{~cm} \mathrm{DBH} \mathrm{[16].} \mathrm{All} \mathrm{of} \mathrm{these} \mathrm{stands} \mathrm{have} \mathrm{been} \mathrm{under}$ attack by both Cryptotoccus fagisuga and Neonectria spp. since the early 1930's [1] and would be considered an aftermath forest. After initial mortality waves, the remaining trees in an aftermath forest are primarily heavily cankered and a lower density persistent scale infestation is present in the stand. Diseased trees were selected along with healthy trees in five of the stands. A healthy tree (greater than $10 \mathrm{~cm} \mathrm{DBH}$ ) and a susceptible tree from Ludington, MI., USA were also included in this study. Beech scale is estimated to have been established in Ludington as early as 1990 and the presence of Neonectria was confirmed in 2001 [11]. At the time of tissue collection, 2004, this was considered a killing front. All diseased trees selected for this study showed visible signs of Neonectria infection such as cankers or the presence of perithecia and scale infestation.

The experimental sampling is unbalanced with respect to disease resistance because the primary interest is in 
resistant genotypes for breeding (more resistant trees are selected for grafting, testing, and planting). Modern statistical algorithms and computer power are sufficient to allow significant imbalance in experiments to be modelled, and we take advantage of this in our experimental design. All trees selected were tested for resistance to the beech scale insect in studies reported previously $[16,65]$ and summarized in Table 1 . These tests demonstrated that all the healthy trees were resistant to the beech scale insect and all of the diseased trees were susceptible to the beech scale insect.

Branches, 1-2 $\mathrm{cm}$ in diameter, were removed from the crown of each of the selected trees in October, 2006 in the New Brunswick stands and in September 2004 in Ludington, MI. Bark was peeled from the branches with a potato peeler and bark strips were placed in labelled $50 \mathrm{~mL}$ falcon tubes, flash frozen and stored in liquid Nitrogen or a dry ice ethanol bath on site. Peeled bark collected from each tree was divided among three tubes and transferred to a $-80^{\circ} \mathrm{C}$ freezer for storage either at the Natural Resources Canada Lab in Fredericton, New Brunswick, Canada or the US Forest Service (USFS) Lab at Delaware, Ohio, USA. In February of 2007, samples from New Brunswick, Canada were shipped overnight on dry ice to Delaware, $\mathrm{OH}$, USA.

\section{Protein extraction}

Protein was extracted according to Bona et al. [66] with minor modifications to account for the high soluble phenolic content of tree bark and phloem tissues. Bark tissue from each tree was combined with dry ice and ground to a course powder in a standard household coffee grinder and then transferred to a $-80^{\circ} \mathrm{C}$ freezer. Three technical replicates were produced from the tissue from each tree. For each replicate, $2 g$ of powdered tissue (after dry ice sublimated off) were combined with $2 \mathrm{~g}$ of frozen polyvinylpolypyrrolidone and $20 \mathrm{~mL}$ of lysis buffer (as per Bona et al. [66] except that 1\% Sigma Plant Proteinase inhibitor cocktail, P-9599, was used in place of $2 \%$ phenylmethylsulfonyl floride/dimethyl sulphoxide) and homogenized using a tissue homogenizer (Janke \& Kunkel, IKA Labortecknik, Ultra-Turrax T25 with $18 \mathrm{~N}$ tip). The resulting homogenate was centrifuged at $26,000 \mathrm{~g}_{\mathrm{n}}$ for 10 minutes at $4^{\circ} \mathrm{C}$ to pellet solids. The supernatant (generally $10 \mathrm{~mL}$ ) was combined with $10 \mathrm{~mL}$ of tris(hydroxymethyl)aminomethane (Tris$\mathrm{HCl}, \mathrm{pH}$ 8.8) saturated phenol and mixed for one hour at room temperature. The phenolic phase was separated by centrifugation and rinsed with another $10 \mathrm{~mL}$ of lysis buffer, followed by further centrifugation to separate the phenolic phase. The final phenolic phase was recovered and proteins were precipitated by adding five volumes of methanol/0.1M ammonium acetate and incubating overnight at $-20^{\circ} \mathrm{C}$. Proteins were pelleted by centrifuging at $26,000 g_{n}$ for 20 minutes and the resulting pellet rinsed three times with cold methanol, once with cold acetone, and dried under vacuum. The pellet was resolubilized in $450 \mathrm{uL}$ of resolubilization buffer (Biorad ReadyPrep sequential extraction reagent II (8M Urea, 4\% 3-[(3-cholamidopropyl) dimethylamonio]-1-propanesulphonate (CHAPS), 40mM Tris, $0.2 \%$ Bio-Lyte $3 / 10$ ampholytes) plus $1 \%$ tris-butyl phosphate (TBP, Sigma T-7567) and 1\% plant proteinase inhibitor cocktail). Proteins were quantified (in triplicate) using the Biorad RC DC protein assay kit (Biorad 500-0118) microfuge tube assay protocol with the optional second wash. Protein quality was checked by running $40 \mu \mathrm{g}$ of protein on a denaturing polyacrylamide gel and staining with coomassie stain as per standard protocols (Biorad mini-protean-3 cell Instruction Manual).

\section{Two-dimensional electrophoresis (2-DE)}

2-DE was conducted at the Plant Microbe Genomics Facility at The Ohio State University (OSU). Isoelectric focusing (IEF) was performed using $11 \mathrm{~cm} \mathrm{pH} \mathrm{3-10} \mathrm{immobilized}$ $\mathrm{pH}$ gradient strips (Biorad, 163-2014) in the Protean IEF Cell (Biorad). For quantitative gels, $100 \mu \mathrm{g}$ of protein was mixed with rehydration buffer (7.0M Urea, 2.0M Thiourea, 2.0\% w/v CHAPS, $2.0 \% \mathrm{w} / \mathrm{v}$ sulfo-betain 10 and focused for $55 \mathrm{kVH}$ at $25^{\circ} \mathrm{C}$. After IEF, the strips were treated according to the ReadyPrep Reduction-Alkylation kit (Biorad, 163-2090) which uses TBP for reduction, and iodoacetamide for alkylation. Second dimension separation was carried out on Criterion 8-16\% Tris- $\mathrm{HCl}$ gels (Biorad 161-1394) using a Criterion Dodeca cell so that all eight gels in the replicate could be run in parallel. Gels were run at $200 \mathrm{~V}$ for 60 minutes and then fixed for 30 minutes in a solution of $10 \%$ methanol and $6 \%$ acetic acid. Gels were then stained with 1x SYPRO-Ruby (Biorad, 170-3138) following manufacturer's instructions. Post staining, gels were de-stained for 1 hour in identical solution as that used for fixation. Preparative gels for spot cutting to recover proteins were prepared in the same way, except that $450 \mu \mathrm{g}$ of protein was used per sample and gels were stained with Coomassie stain (Biorad, 161-0787) following manufacturer's instructions.

\section{Image analysis and quantification}

Gels were imaged on a VersaDoc imager (Biorad), and the software program PDQuest (version 8.0, Biorad) was used to conduct the image analysis, spot identification and quantification. Gel images were digitally cropped along the outer edge to remove the molecular size marker and gel edges, and to standardize image size, but both pI fronts and the full size resolving area were retained.

The spot selection and gel matching were conducted in two stages, first a separate master gel was created for each tree by auto-matching the three replicate gels using the 'create experiment' dialog boxes of PDQuest. For these tree master gels, the spot detection and automated 
spot matching are conducted as part of the same procedure. For spot detection we used the spot detection wizard with vertical streak reduction on, and selecting the user chosen reference spot for small spot, faint spot, and large spot cluster from the same region of the gel for all gels. In addition we selected the local area regression method of normalization, which is proprietary but appears to be based on similar microarray normalization methods (see Quackenbush [67]). For spot matching, we defined no groups and spots were added to the master image only if present in two of three gels. Auto-matched spots were manually checked and corrected by dividing the gel area into 81 quadrants and hand marking landmark spots in each quadrant present in all three gels. All of the matches were hand checked based upon these landmark spots, and manual corrections to the spot detection and auto-matching were made, including removal of spots detected on the unresolved $\mathrm{pI}$ fronts and gel edges.

The second phase of image analysis was to create a 'compare experiments' analysis including all sixteen individual tree 'master gels' (CM02d was software selected as the base master gel). Automated matching was used to create the initial master file, then all matches were manually checked. Additional spots were added to the master manually if they were present in two or more tree masters. We applied the same hand check quality control as for individual tree masters (manually checking and correcting each of 81 quadrants using landmark spots) and applied the same normalization method (local area regression model option). Of note, we did not incorporate an additional scaling factor and the normalization method doesn't scale the data, so the final spot quantities still have the original unit of counts.

Once the compare experiment master gel was fully checked, a quantitative dataset was created. The quantitative dataset was output from PDQuest using the function Report| Quantity Table Report, with the settings: all matched spots checked, configuration set to 'individual gels', missing spots set to 'estimate', and saturated spots set to 'estimate'. Spot quantities were estimated so that analysis options that require balanced and nonzero datasets could be used. PDQuest estimates saturated spots by fitting a Gaussian spot to the edges only and extrapolating the peak, then calculating the estimated volume from the extrapolated value. PDQuest estimates missing spots as the value of a minimum detectable spot. The resulting report contained spot quantities for all spots in the master gel across all 48 experimental gels. Graphical analysis of the spot quantities by spot were deemed sufficiently normally distributed to proceed with modelling.

To be sure the unmatched spots that are unique to one tree were not artifacts related to low spot intensity or variance in protein quantification making it difficult to match them, a random check of the intensity distribution of unmatched spots was conducted. Four trees were selected at random, then the spot intensity output for all spots on the tree master for these four trees was examined both visually by inspecting spot intensities in the gels and by comparing the distribution of intensities of the unique spots to the matched spots. Comparisons were made both graphically and by using summary statistics (mean, minimum, Q1, median, Q3, and maximum values).

\section{Experimental design and statistical analysis}

The experiment included 16 trees, each of which had a location (stand) code and a disease condition code. Three replicate extractions (for three separate gels) were run per tree. For each replicate there were a total of 16 extractions, one per tree. Each protein extraction was assigned first to one of four extraction sets (three healthy and one diseased tree per set), then extraction sets paired to form gel sets (two gel sets per replicate). Samples in an extraction set were extracted in parallel, and gel sets were run in parallel for both IEF and polyacrylamide gel separation. Hence, each extraction had a full list of factors assigned: tree, stand, disease state, replicate, extraction set, gel set. The full dataset included these factors and spot quantities for each spot on the master gel for each of the 48 gels (see additional files: Additional file 2, full study design; Additional file 3, full set of spot quantities). Calculated spot quantities were from the normalized gel images, and were evaluated and determined to need no additional transformation. An ANOVA approach to statistical analysis was used to so that multiple effects and interactions could be included in the same model to better control error variance, and because the other biological effects (i.e. stand and its interaction with $\mathrm{BBD}$ ) will be informative in selecting proteins for future study.

Statistical analysis was generated using SAS $^{\circledR}$ software version 9.2 of the SAS system for Windows, copyright 2002-2008 SAS Institute Inc. SAS and all other SAS Institute Inc. products or service names are registered trademarks or trademarks of SAS Institute, Cary, NC, USA.

A series of tests were used to categorize each protein spot, to arrive at a list of candidate spots for further analysis (summarized in Figure 3). The first phase of the analysis sought to exclude constitutive proteins that did not differ between any trees, and assess the significance of the technical factors. The following model was fit using the General Linear Model procedure of SAS:

$$
\text { intensity }=\mu+t_{j}+g_{k(1)}+\varepsilon_{\mathrm{ijk}(1)}
$$

where $\mu$ is an overall average, $t_{j}$ is the effect of the $j^{\text {th }}$ tree (tree effect), $g_{k(1)}$ is the effect of the $l^{\text {th }}$ extraction set nested within the $\mathrm{k}^{\text {th }}$ gel set (technical effect), and $\varepsilon_{\mathrm{ijk}(\mathrm{l})}$ is a random error term. The model was fit for each 
spot, and the test of significant effects computed using the type III sums of squares. Model fit was evaluated by verifying that the overall model fit had a significant $F$ value ( $p$-value $<0.05$ ) and by examination of standardized residuals (especially by plotting against the levels of effects). For each model, careful assessment of residual plots confirmed model assumptions about error distribution and equal variances were sufficiently met (i.e. the spot quantities did not deviate from the assumed normal distribution enough to warrant additional transformation). Degrees of freedom (df) were the same for each spot model: tree effect has $15 \mathrm{df}$, technical effect had $11 \mathrm{df}$, and error $\mathrm{df}=21$ (total $\mathrm{df}=47$ ). For the technical effect, a Bonferroni adjustment was used to determine significance level, but for the tree effect a $\mathrm{p}<=0.05$ was considered significant. This permissive cut off is appropriate since the goal of the test was to eliminate constitutive proteins (and reduce the physical size of the dataset to ease computations) and because a false acceptance of the null is more problematic than a false rejection at this point in the analysis. Any spots that are not significantly different in at least one tree (tree effect $\mathrm{p}<0.05$ ) were dropped from the dataset. Technical effects were found to be not significant and were dropped from further analysis for all but six spots that were dropped from the dataset.

The second phase of the analysis was designed to determine how spots differed between trees. Technical factors were dropped and stand and disease state factors were added (there were insufficient degrees of freedom to analyse technical and biological factors in the same model, partly due to imbalance in the number of trees selected per stand). The following model was fit using the General Linear Model procedure of SAS:

$$
\text { intensity }=\mu+s_{j}+d_{k}+\operatorname{sd}_{\mathrm{jk}}+\varepsilon_{\mathrm{ijk}}
$$

where $\mu$ is an overall average, $s_{j}$ is the effect of the $j^{\text {th }}$ stand (stand effect), $d_{k}$ is the effect of the $k^{\text {th }}$ disease state (BBD effect), $s d_{j k}$ is the interaction effect of stand and disease state, and $\varepsilon_{\mathrm{ijk}}$ is a random error term. Model fit was evaluated by verifying that the overall model fit had a significant $F$ value ( $p$-value $<0.05$ ) and by examination of standardized residuals (especially by plotting against the levels of effects). For each model, careful assessment of residual plots confirmed model assumptions about error distribution and equal variances were sufficiently met (i.e. the spot quantities did not deviate from the assumed normal distribution enough to warrant additional transformation). Degrees of freedom were the same for each spot model: BBD effect has 1df, STAND effect has $7 \mathrm{df}$, BBDxSTAND interaction effect has $5 \mathrm{df}$, and error has $34 \mathrm{df}$ (total $\mathrm{df}=47$ ). The model was fit for each spot, and the test of significant effects computed using the type III sums of squares. Interaction effects and stand effect were tested using the conservative Bonferroni correction. For the BBD effect, p-values from the tests were output to a new dataset and the package 'qvalue' (q-value 1.1) for the statistical program R (R-2.4.0win32, [68]) was used to compute the associated q-value for each test. Significance was determined using q-values while controlling the false discovery rate at 5\% [69]. False discovery rate controls the percentage of null hypothesis rejected in error (false positives) rather than the overall error rate, and is an accepted and typical statistical analysis for large genomic and proteomic datasets [70].

\section{Spot selection and cutting}

All spots with a significant effect for the disease state factor were considered for spot cutting and sequencing. Spot quantities were evaluated in all of the trees and trees ranked as the best trees were those having the most BBD significant spots at the highest spot densities. The two top trees were used for preparative gels and spot cutting. All BBD significant spots in the two selected trees were evaluated on the gel images to determine if the spot could be excised cleanly and was sufficiently intense to support sequencing. Spots were excised from the preparative gels at the PMGF using the Protean 2-D spot cutter (Biorad Laboratories). Several constitutive spots were also selected as sequencing reference spots. High resolution pre-cut and post-cut images of preparative gels were captured on the VersaDoc imager and evaluated for quality control. Only protein spots that were cleanly excised and had no evidence of contamination from adjacent spots were sent for MS/MS analysis.

\section{Mass spectrometry}

Mass spectrometry was performed at the OSU Campus Chemical Instrumentation Center. Gel pieces were washed twice in $50 \%$ methanol $/ 5 \%$ acetic acid for one hour each, followed by dehydration in acetonitrile. Cysteines were reduced by rehydrating and incubating in dithiothreitol (DTT) solution $(5 \mathrm{mg} / \mathrm{mL}$ in $100 \mathrm{mM}$ ammonium bicarbonate) for 30 minutes. Cysteins were alkylated by the addition of $15 \mathrm{mg} / \mathrm{mL}$ iodoacetamide in 100 $\mathrm{mM}$ ammonium bicarbonate solution, and incubation in the dark for $30 \mathrm{~min}$. The gel cores were washed again with cycles of acetonitrile and ammonium bicarbonate $(100 \mathrm{mM})$ in $5 \mathrm{~min}$ increments, then dried under vacuum. Protein was digested in Multiscreen Solvinert Filter Plates from Millipore (Bedford, MA) with sequencing grade modified trypsin (Promega, Madison WI) overnight. The peptides were extracted from the polyacrylamide by washing several times with $50 \%$ acetonitrile and $5 \%$ formic acid, pooled, and concentrated under vacuum to $\sim 30 \mathrm{uL}$. 
Capillary-liquid chromatography-nanospray tandem mass spectrometry (Nano-LC/MS/MS) was performed on a Thermo Finnigan LTQ mass spectrometer equipped with a nanospray source operated in positive ion mode. The LC system was an UltiMate ${ }^{\mathrm{TM}} 3000$ system from Dionex (Sunnyvale, CA). Five microliters of each sample were first injected on to the micro-Precolumn Cartridge (Dionex, Sunnyvale, CA), and washed with $50 \mathrm{mM}$ acetic acid. The injector port was switched to inject and the peptides were eluted off of the trap onto the column. A $5 \mathrm{~cm} 75 \mu \mathrm{m}$ ID ProteoPep II C18 column (New Objective, Inc. Woburn, MA) packed directly in the nanospray tip was used for chromatographic separations. Peptides were eluted directly off the column into the LTQ system using a gradient of $2-80 \%$ acetonitrile over 45 minutes, with a flow rate of $300 \mathrm{nl} / \mathrm{min}$ and total run time was 65 minutes. The MS/MS was acquired using a nanospray source operated with a spray voltage of $3 \mathrm{KV}$ and a capillary temperature of $200^{\circ} \mathrm{C}$. The analysis was programmed for a full scan recorded between $350-2000$ $\mathrm{Da}$, and a MS/MS scan to generate product ion spectra to determine amino acid sequence in consecutive instrument scans of the ten most abundant peaks in the spectrum. The CID fragmentation energy was set to $35 \%$. Dynamic exclusion is enabled with a repeat count of 30s, exclusion duration of 350s and a low mass width of $0.5 \mathrm{Da}$ and high mass width of $1.50 \mathrm{Da}$.

\section{Sequence data processing and matching}

Sequence information from the MS/MS data were searched using Mascot Daemon (version 2.2.1 Matrix Scientific, Boston, MA) [71] against several databases (detailed below). The search parameters were: mass accuracy of the precursor ions $=2.0$, fragment mass accuracy $=0.5 \mathrm{Da}$, considered (variable) modifications = methionine oxidation and carbamidomethyl cysteine, missed cleavages $=2-4$. Due to the low representation of woody plant and bark tissue sequences in the databases, the search was conducted against several databases. Searching against the full SwissProt database version 54.1 (283454 sequences; 104030551 residues) was unproductive (only procedural peptides identified, data not shown). A second search was conducted restricting the search set to taxon Viridiplantae (version, sequences, residues). The Fagaceae genomics project [72] has constructed EST libraries from American Beech, Red Oak (Quercus rubra L.), White Oak (Quercus alba L.), American chestnut (Castanea dentata (Marsh.) Borkh) and Chinese chestnut (Castanea mollissima Blume) including libraries constructed from both healthy and diseased stem tissues. Twenty-four individual EST libraries (\#13696, 8-21-2009, 10691208 sequences; 751178460 residues) were compiled into a custom database and searched. Peptide matches were checked manually and only those identifications with a Mascot score of 50 or higher and having two or more unique peptides of five or more residues were accepted. For EST matches, peptides were matched to EST's (by the same criteria), then EST's searched against GenBank (BLASTP, default settings [73] to obtain protein identifications. Analysis data is available in the PRIDE database [74,75] under the accession numbers 17706. The data was converted using the PRIDE Converter [76,77].

\section{Additional files}

Additional file 1: p-values from ANOVA models. Complete set of $p$-values under-laying Table 3 including the initial $p$-value and the computed q-values and determination of significance based on q-value or Bonferroni correction.

Additional file 2: Full experimental design. The full design of the experiment by gel, including randomization to extraction batch and gel batch.

Additional file 3: Full set of spot quantities. Spread sheet of the full spot quantifications for 48 gels showing the value of counts for each spot on each gel.

\section{Abbreviations}

2-DE: 2 Dimensional Electrophoresis; ANOVA: Analysis Of Variance; BBD: Beech Bark Disease, or its effect in a statistical model; CHAPS: 3-[(3Cholamidopropyl)Dimethylamonio]-1-Propanesulphonate; DF: Degrees of Freedom in a statistical model or test; IEF: Iso-Electric Focusing; INT: The Interaction Effect (Bbdxstand) In Statistical Models; OSU: The Ohio State University; STAND: The Effect Of Stand In The Statistical Models; TBP: TrisButyl Phosphate; Tris-HCl: Tris(Hydroxymethyl)Aminomethane.

\section{Competing interests}

We would like to acknowledge and declare several funding sources: Parks Canada Ecological Integrity Innovation and Leadership Fund, Fundy Model Forest, Canadian Forest Service, Northern Research Station of the US Forest Service, and the Special Technology Development Program of the Northeast Area State and Private Forestry, US Forest Service.

\section{Authors' contributions}

MEM carried out molecular experiments, statistical analysis, and drafted the manuscript under the supervision of JLK who helped design the study and draft the manuscript. JLK, JL and MK developed the study concept and carried out field and artificial infestation experiments. All authors read and approved the final manuscript.

\section{Acknowledgements}

We would like to thank Donnie McPhee and Kathleen Forbes for their assistance with collecting material, Tannis Beardmore for practical advice, and The Fagaceae Genomics Tools project [72] for access to EST data from American Beech. We thank John Carlson, Sasha Popova-Butler, and Sourav Chakraborty for helpful comments on a draft version of this manuscript. And we are grateful to Dave Carey for his excellent technical work and advice on all aspects of the beech bark disease research project.

\section{Author details}

'Department of Entomology, Ohio Agricultural Research and Development Center, The Ohio State University, 1680 Madison Ave, Wooster, OH 44691, USA. ${ }^{2}$ US Forest Service, Northern Research Station, 359 Main Rd, Delaware, $\mathrm{OH} 43015$, USA. ${ }^{3}$ Faculty of Forestry and Environmental Management, University of New Brunswick, P.O. Box 440028 Dineen Drive, Fredericton, NB E3B 5A3, Canada. ${ }^{4}$ Formerly: Canadian Forest Service, Natural Resources Canada, P.O. Box 4000, Fredericton, NB E3B 6J6, Canada. ${ }^{5}$ Present address: Bioversity International, Via dei Tre Denari, 472/a, 00057 Maccarese, Rome, Italy. 
Received: 9 May 2012 Accepted: 23 December 2012

Published: 14 January 2013

\section{References}

1. Ehrlich J: The beech bark disease of Fagus, following crytococcus fagi (Baer.). Can J Res 1934, 10:593-692.

2. Houston DR: Major new tree disease epidemics: beech bark disease. Ann R Phytopathol 1994, 32:75-87.

3. Miller-Weeks M: Current status of beech bark disease in New England and New York. In Proceedings, IUFRO beech bark disease working party conference: Sept 26-Oct 8, 1982; Hamden CT. Gen. Tech. Rep. WO-37. Edited by Houston DR, Wainehouse E. Washington, DC: USDA Forest Service, Northeastern Forest Experiment Station; 1983:21-23.

4. Houston DR: American beech resistance to Cryptococcus fagisuga. In Proceedings, IUFRO beech bark disease working party conference: Sept 26-Oct 8, 1982; Hamden CT. Gen. Tech. Rep. WO-37. Edited by Houston DR, Wainehouse E. Washington, DC: USDA Forest Service, Northeastern Forest Experiment Station; 1983:38-41.

5. Koch JL, Carey DW, Mason ME, Nelson CD: Assessment of beech scale resistance in full- and half-sibling American beech families. Can J For Res 2010, 40:265-272.

6. Mackenzie M: Observations of the beech bark disease complex on the national forests of Pennsylvania and West Virginia. In Proceedings of the 53rd western international forest disease work conference. Aug. 26-29, 2005. Edited by Jackson WY. Ogden, UT: Compiled by Guyon, J. US Forest Service, Forest Health Protection; 2006:31-38.

7. Garnas JR, Houston DR, Ayres MP, Evans C: Disease ontogeny overshadows effect of climate and species interactions on population dynamics in a non-native forest disease complex. Ecography 2012, 35:412-421.

8. Kasson MT, Livingston WH: Relationships among beech bark disease climate, radial growth response and mortality of American beech in northern Maine USA. Forest Pathology 2012, 42:199-212.

9. Cale JA, Letkowski SK, Teale SA, Castello JD: Beech bark disease: an evaluation of the predisposition hypothesis in an aftermath forest. Forest Pathology 2012, 42:52-56.

10. Koch JL: Beech bark disease: the oldest "new" threat to American beech in the united states. Outlooks on Pest Management 2010, 21:64-68.

11. Heyd R: Managing beech bark disease in Michigan. In Beech bark disease: proceedings of the beech bark disease symposium. June 16-18, 2004; Saranac lake NY. Gen. Tech. Rep. NE-33. Edited by Evans CA, Jucas JA, Twery MJ. Newtown Square, PA: USDA Forest Service, Northeastern Research Station; 2005:128-132

12. Leak WB, Gove JH: Growth of northern hardwoods in New England: a 25year update. North J Appl For 2008, 25:103-105.

13. US forest service, Allegheny national forest. http://www.fs.usda.gov/Internet/ FSE_DOCUMENTS/stelprdb5044088.pdf.

14. State of Michigan, department of natural resources. http://fhm.fs.fed.us/fhh/ fhh_11/ML_FHH_2011.pdf.

15. Wargo PM: Amino nitrogen and Phenolic constituents of bark of American beech, Fagus grandifolia, and infestation by beech scale Cryptococcus fagisuga. Eur J For Path 1988, 18:279-290.

16. Ramirez M, Loo J, Krasowski M: Evaluation of genetic resistance to the beech scale insect (Cryptococcus fagisuga) and propagation of American beech (Fagus grandifolia) by grafting. Silvae Genet 2007, 56:163-169.

17. Li L, Steffens JC: Over-expression of polyphenol oxidase in transgenic tomato plants results in enhanced bacterial disease resistance. Planta 2002, 215:239-247.

18. Thipyapong P, Hunt MD, Steffens JC: Antisense down-regulation of polyphenol oxidase results in enhanced disease susceptibility. Plant 2004, 220:105-117.

19. Ramiro DA, Guerreiro-Filho O, Mazzafera P: Phenol contents, oxidase activities, and the resistance of coffee to the leaf miner Leucoptera coffeella. J Chem Ecol 2006, 32:1977-1988.

20. Bustos DM, Bustamante CA, Iglesias AA: Involvement of nonphosphorylating glyceraldehydes-3-phosphate dehydrogenase in response to oxidative stress. J Plant Physiol 2008, 165:451-461.

21. Baek D, Jin Y, Jeong JC, Lee H, Moon H, Lee J, Shin D, Kang CH, Kim DH, Nam J, Lee SY, Yun D: Suppression of reactive oxygen species by glyceraldehydes-3-phosphate dehydrogenase. Phytochemistry 2008, 69:333-338.
22. Hancock JT, Henson D, Nyirenda M, Desikan R, Harrison J, Lewis M, Hughes J, Neill SJ: Proteomic identification of glyceraldehydes-3-phosphate dehydrogenase as an inhibitory target of hydrogen peroxide in Arabidopsis. Plant Physiol Biochem 2005, 43:828-835.

23. Collins RM, Afzal M, Ward DA, Prescott MC, Sait SM, Rees HH, Tomsett AB: Differential proteome analysis of Arabidopsis thaliana genotypes exhibiting resistance or susceptibility to the insect herbivore Plutella xylostella. PLoS One 2010, 5:1-14.

24. Monstyrskaya K, Babiychuk EB, Draeger A: The annexins: spatial and temporal coordination of signaling events during cellular stress. Cell Mol Life Sci 2009, 66:2623-2642.

25. Mortimer JC, Laohavisit A, Macpherson N, Webb A, Brownlee C, Battey NH, Davies JM: Annexins: multifunctional components of growth and adaptation. J Exp Bot 2008, 59:533-544.

26. Konupka-Postupolska D, Clark G, Goch G, Debski J, Floras K, Canero A, Fijolek B, Roux S, Hennig J: The role of annexin 1 in drought stress in Arabidopsis. Plant Physiol 2009, 150:1394-1410

27. Montrichard F, Alkhalfioui F, Yano J, Vensel WH, Hurkman WJ, Buchanan BB: Thioredoxin targets in plants: the first 30 years. J Proteomics 2009, 72:45-474.

28. Lalo C, Mestres-Ortega D, Marco Y, Meyer Y, Reichheld J: The Arabidopsis cytosolic thioredoxin h5 gene induction by oxidative stress and its W-box-mediated response to pathogen elicitor. Plant Physiol 2004, 134:1006-1016.

29. Park SK, Jung YJ, Lee JR, Lee YM, Jang HH, Lee SS, Park JH, Kim SY, Moon JC, Lee SY, Chae HB, Shin MR, Jung JH, Kim MG, Kim WY, Yun D, Lee KO, Lee SY: Heat-shock and redox-dependent functional switching of an h-type Arabidopsis thioredoxin from a disulfide Reductase to a molecular chaperone. Plant Physiol 2009, 150:552-561.

30. Traverso JA, Vignols F, Cazalis R, Pulido A, Sahrawy M, Cejudo FJ, Meyer JC, Chueca A: PsTRXh1 And PsTRXh2 are both pea h-type thioredoxins with antagonistic behavior in redox imbalances. Plant Physiol 2007, 143:300-311.

31. Close TJ: Dehydrins: emergence of a biochemical role for a family of plant dehydration proteins. Physiol Plant 1996, 97:795-803.

32. Rorat T: Plant dehydrins - tissue localization, structure and function. Cell mol biol Itrs 2006, 11:536-556.

33. Ventelon-Debout M, Delalande F, Brizard J, Diemer H, Dorsselaer AV, Brugidou C: Proteome analysis of cultivar-specific deregulations of Oryza sativa indica and O.sativa japonica cellular suspensions undergoing rice yellow mottle virus infection. Proteomics 2004, 4:216-225.

34. Gang DR, Kasahara H, Zia Z, Mijnsbrugge KV, Bauw G, Boerjan W, Montagus MV, Davin LB, Lewis NG: Evolution of plant defense mechanisms. J Biol Chem 1999, 11:7516-7527.

35. Manosalvo PM, Davidson RM, Liu B, Zhu X, Hulbert SH, Leung H, Leach JE: A germin-like protein gene family functions as a complex quantitative trait locus conferring broad-spectrum disease resistance in rice. Plant Physiol 2009, 149:286-296

36. Zhou S, Sauve R, Thannhauser TW: Proteome changes induced by aluminium stress in tomato roots. J Exp Botany 2009, 6:1849-1857.

37. Uzarowska A, Dionisio G, Sarholz B, Piepho H, Xu M, Ingvardsen CR, Wenzel $\mathrm{G}$, Lubberstedt T: Validation of candidate genes putatively associated with resistance to SCMV and MDMV in maize (Zea Mays L.) by expression profiling. BMC Plant Biol 2009, 9:15-30.

38. Soares NC, Francisco R, Vielba JM, Ricardo CP, Jackson PA: Associating wound-related changes in the apoplast proteome of Medicago with early steps in the ROS signal-transduction pathway. J Proteome Res 2009, 8:2298-2309.

39. Park C, An J, Shin Y, Kim K, Lee B, Pack K: Molecular characterization of pepper germin-like protein as the novel PR-16 family of pathogenesisrelated proteins isolated during the resistance response to viral and bacterial infection. Planta 2004, 219:797-806.

40. Mitchell SF, Lorsch JR: Should I stay or should I go? eukaryotic translation initiation factor 1 and $1 \mathrm{~A}$ control start codon recognition. J BiolChem 2008, 283:27345-27349.

41. Raussell A, Kanhonou R, Yenush L, Serrano R: The translation initiation factor elR1A is an important determinant in the tolerance to $\mathrm{NaCl}$ stress in yeast and plants. Plant J 2003, 34:257-267.

42. Scmidt F, Marnef A, Cheung M, Wilson I, Hancock J, Staiger D, Ladomery M: A proteomic analysis of oligo(dT)-bound mRNP 
containing oxidative stress-induced Arabidpopsis thaliana RNAbinding proteins ATGRP7 and ATGRP8. Mol Biol Rep 2010, 37:839-845.

43. Lee M, Kim LP, Kim B, Hahn J, Hong CB: Flooding stress-induced Glycine-rich RNA-binding protein from Nicotiana tabacum. Mol Cells 2009, 27:47-54.

44. Rospert S, Dubaquie Y, Gautschi M: Nascent-polypeptide-associated complex. Cell Mol Life Sci 2002, 59:1632-1639.

45. Yan S, Tang Z, Su W, Sun W: Proteomic analysis of salt stress-responsive proteins in rice root. Proteomics 2005, 5:235-244.

46. Hakkanen A, Kokko H, Buchala A, Hayrinen J, Karenlampi S: Benzothiadiazole affects the leaf proteome in arctic bramble (Rubus arcticus). Mol Plant Pathol 2008, 9:799-808.

47. Lee $Y$, Lee H, Lee J, Kim S, Kim S: Hormone- and light-regulated nucleoctyoplasmic transport in plants: current status. J Exp Bot 2008 59:3229-3245

48. Subramanian B, Bansa VK, Kav NNV: Proteome-level investigation of Brassica carinata-derived resistance to leptosphaeria maculans. J Agric Food Chem 2005, 53:313-324

49. He H, Li J: Proteomic analysis of phosphoproteins regulated by abscisic acid in rice leaves. Biochem Biophys Res Comm 2008, 371:883-888.

50. Kushwaha HR, Singh AK, Sopry SK, Singla-Pareek S, Pareek A: Genome wide expression analysis of CBS domain containing proteins in Arabidopsis thaliana (L.) heynh and Oryza sativa L. Reveals their developmental and stress regulation. BMC Genomics 2009, 10:200.

51. Abril N, Gion JM, Kerner R, Muller-Starck G, Navarro Cerrillo RM, Plomion C, Renaut J, Valledor L, Jorrin-Novo JV: Proteomics research on forest trees, the most recalcitrant and orphan plant species. Phytochemistry 2011, 72:1219-1242.

52. Koch JL, Carey DW: Controlled cross-pollinations with American beech trees that are resistant to beech bark disease. In Proceedings, 14th central hardwood forest conference: march 16-19, 2004; Wooster OH. Gen. Tech. Rep. NE-316. Edited by Yaussy DA, Hix DM, Long RP, Geobel PG. Newtown Square, PA: USDA Forest Service, Northeastern Research Station; 1984:358-364.

53. Koch JL, Carey DW, Mason ME: Use of microsatellite markers in an American beech (Fagus grandifolia) population and paternity testing. Silvae Genet 2009, 9:62-68.

54. Bahrman N, Zivy M, Damerval C, Baradat P: Organisation of the variability of abundant proteins in seven geographical origins of maritime pine (Pinus pinaster Ait.). Theor Appl Genet 1994, 88:407-411.

55. Kunkel BN, Brooks DM: Crosstalk between signalling pathways in pathogen defense. Curr Opin Plant Biol 2002, 5:325-331.

56. Zhang $E_{1}$ Chen Z, Liang X: Resolubilization of TCA precipitated plant proteins for 2-D electrophoresis. Electrophoresis 2011, 32:696-698.

57. Zhu Y, Zhao P, Wu X, Wang W, Scali M, Cresti M: Proteomic identification of differentially expressed proteins in mature and germinated maize pollen. Acta Physio Plant 2011, 33:1467-1474.

58. Zhao F, Fang W, Xie D, Zhao Y, Tang Z, Li W, Nie L, Lv S: Proteomic identification of differentially expressed proteins in Gossypium thurberi inoculated with cotton Verticulum dahliae. Plant Sci 2012, 185-186:176-184.

59. Mohammadi PP, Moieni A, Hiraga S, Komatsu S: Organ-specific proteomic analysis of drought-stressed soybean seedlings. J Proteomics 2012, 75:1906-1923.

60. Bai S, Willard B, Chapin LJ, Kinter MT, Francis DM, Stead AD, Jones ML: Proteomic analysis of pollination-induced corolla senescence in petunia. $J$ Exp Bot 2010, 61:1089-1109.

61. Kerner R, Winkler JB, Dupuy JW, Jurgensen M, Lindermayr C, Ernst D, MullerStarck G: Changes in the proteome of juvenile European beech following three years exposure to free-air ozone. iForest 2011, 4:69-76.

62. Pawlowski TA: Proteomics of European beech (Fagus sylvatica L.) seed dormancy breaking: influence of abscisic and gibberellic acids. Proteomics 2007, 7:2246-2257.

63. Valcu C, Junqueira M, Shevchenko A, Schlink K: Comparative proteomic analysis of responses to pathogen infection and wounding in Fagus sylvatica. J Prot Res 2009, 8:4077-4091.

64. Morrin RS, Liebhold AM, Luzader ER, Lister AJ, Gottschalk KW, Twardus DB: Mapping host-species abundance of three major exotic forest pests. Res. Pap. NE-726. Newtown Square, PA: USDA Forest Service, Northeastern Research Station; 2005:11.

65. Koch $J$, Carey DW: The genetics of resistance of American beech to beech bark disease: knowledge through 2004. In Beech bark disease: proceedings of the beech bark disease symposium. June 16-18, 2004; Saranac lake NY. Gen. Tech. Rep. NE-331. Edited by Evans CA, Jucas JA, Twery MJ. Newtown Square, PA: USDA Forest Service, Northeastern Research Station; 2005:98-105.

66. Bona E, Marsano F, Cavaletto M, Berta G: Proteomic characterization of copper stress response in Cannabis sativa roots. Proteomics 2007 7:1121-1130.

67. Quackenbush J: Microarray normalization and transformation. Nat Genet 2002, 32(Suppl):496-501.

68. 18 The R project website. http://www.r-project.org

69. Storey JD: Strong control, conservative point estimation and simultaneous conservative consistency of false discovery rate: a unified approach. J R Statist Soc B 2004, 1:187-205.

70. Gutstein HB, Morris JS: Laser capture sampling and analytical issues in proteomics. Exp Rev Proteomics 2007, 4:627-637.

71. Perkins DN, Pappin DJC, Creasy DM, Cottrell JS: Probability-based protein identification by searching sequence databases using mass spectrometry data. Electrophoresis 1999, 20:3551-3567.

72. The Fagaceae genomic project website. www.fagaceae.org

73. Altshcul SF, Madden TL, Schäffer AA, Zhang J, Zhang Z, Miller W, Lipman DJ: Gapped BLAST and PSI-BLAST: a new generation of protein database search programs. Nuc Acids Res 1997, 25:3389-3402.

74. Vizcaino JA, Cote R, Reisinger F, Barnes H, Foster JM, Rameseder J, Hermjakob H, Martens L: The proteomics identifications database: 2010 update. Nuc Acids Res 2010, 38:D736-D742.

75. The pride database website. www.ebi.ac.uk/pride.

76. Barsnes H, Vizcaino JA, Eidhammer I, Martens L: PRIDE converter: making proteomics data-sharing easy. Nat Biotechnol 2007, 27:598-599.

77. The pride converter website. http://code.google/p/pride-converter.

doi:10.1186/1477-5956-11-2

Cite this article as: Mason et al:: Comparisons of protein profiles of beech bark disease resistant and susceptible American beech (Fagus grandifolia). Proteome Science 2013 11:2

\section{Submit your next manuscript to BioMed Central and take full advantage of:}

- Convenient online submission

- Thorough peer review

- No space constraints or color figure charges

- Immediate publication on acceptance

- Inclusion in PubMed, CAS, Scopus and Google Scholar

- Research which is freely available for redistribution 